Pacific Journal of Mathematics

SPACES DETERMINED BY POINT-COUNTABLE COVERS

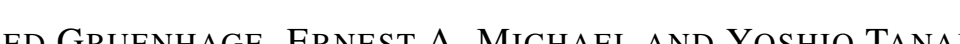




\title{
SPACES DETERMINED BY POINT-COUNTABLE COVERS
}

\author{
G. Gruenhage, E. Michael and Y. TanaKa
}

\begin{abstract}
Recall that a collection $\mathcal{P}$ of subsets of $X$ is point-countable if every $x \in X$ is in at most countably many $P \in \mathcal{P}$. Such collections have been studied from several points of view. First, in characterizing various kinds of $s$-images of metric spaces, second, to construct conditions which imply that compact spaces and some of their generalizations are metrizable, and finally, in the context of meta-Lindelöf spaces. This paper will make some contributions to all of these areas.
\end{abstract}

1. Introduction. A space $^{1} X$ is determined by a cover $\mathscr{P}$, or $\mathscr{P}$ determines $X,{ }^{2}$ if $U \subset X$ is open (closed) in $X$ if and only if $U \cap P$ is relatively open (relatively closed) in $P$ for every $P \in \mathcal{P}$. (For example, $X$ is determined by $\mathscr{P}$ if $\mathcal{P}$ is locally finite or if $\left\{P^{0}: P \in \mathcal{P}\right\}$ covers $X$.) We will explore this notion in the context of point-countable covers.

Within the class of $k$-spaces, spaces determined by point-countable covers turn out to be related to spaces having a point-countable $k$-network. Recall that a cover $\mathscr{P}$ of $X$ is a $k$-network for $X$ if, whenever $K \subset U$ with $K$ compact and $U$ open in $X$, then $K \subset \cup \mathscr{F} \subset U$ for some finite $\mathscr{F} \subset \mathcal{P}$. Such collections have played a role in $\aleph_{0}$-spaces (i.e., regular spaces with a countable $k$-network $\left.\left[\mathbf{M}_{1}\right]\right)$ and $\aleph$-spaces (i.e., regular spaces with a $\sigma$-locally finite $k$-network [0]). ${ }^{3}$

Diagram I below gives a convenient overview of some of our principal results. The numbered conditions are defined as follows, where in (1.3) and elsewhere in this paper we adopt the convention that, if $\mathcal{Q}$ is a collection of sets, then $\mathbb{Q}^{*}$ denotes $\{\cup \mathscr{F}: \mathscr{F} \subset \mathbb{Q}, \mathscr{F}$ finite $\}$.

(1.1) $X$ has a point-countable cover $\mathscr{P}$ such that each open $U \subset X$ is determined by $\{P \in \mathcal{P}: P \subset U\}$.

\footnotetext{
'All spaces are assumed to be Hausdorff.

${ }^{2}$ We use " $X$ is determined by $\mathcal{P}$ " instead of the usual " $X$ has the weak topology with respect to $\mathscr{\rho}$ ". The term "weak topology" is used in a different way by functional analysts, to whom it implies that $X$ has the smallest (not the largest) topology that gives each member of $\mathcal{P}$ its subspace topology. Our terminology avoids this problem, and is also shorter.

${ }^{3}$ While the elements of the defining $k$-network may always be chosen closed in $\boldsymbol{\aleph}_{0}$-spaces and in $\aleph$-spaces (by simply taking closures), that is not always true for point-countable $k$-networks (see Example 9.2).
} 
(1.2) $X$ has a point-countable cover $\mathscr{P}$ such that, if $x \in U$ with $U$ open in $X$, then $x \in(\cup \mathcal{F})^{0} \subset \cup \mathcal{F} \subset U$ for some finite $\mathscr{F} \subset \mathcal{P}$.

(1.2) $)_{p}$ Same as (1.2) with $U=X \backslash\{p\}$ for some $p \in X$.

(1.3) $X$ has a point-countable cover $\mathscr{P}$ such that each open $U \subset X$ is determined by $\{P \in \mathscr{P}: P \subset U\}^{*}$.

(1.3) Same as (1.3) with $U=X \backslash\{p\}$ for some $p \in X$.

(1.4) $X$ has a point-countable $k$-network.

$(1.4)_{p} X$ has a point-countable cover $\mathscr{P}$ such that, if $K \subset U$ is compact and $p \in X \backslash K$, then $K \subset \cup \mathscr{F} \subset X \backslash\{p\}$ for some finite $\mathscr{F} \subset \mathcal{P}$.

(1.5) $X$ has a point-countable cover $\mathscr{P}$ such that, if $x \in K \subset U$ with $K$ compact and $U$ open in $X$, then there is a finite $\mathscr{F} \subset \mathcal{P}$ such that $\cup \mathscr{F} \subset U$, $x \in \cap \mathscr{F}$, and $\mathscr{F}$ covers a neighborhood of $x$ in $K$.

(1.6) $X$ has a point-countable closed $k$-network.

It should be remarked that conditions (1.2) and (1.2) $)_{p}$ were studied in $\left[\mathbf{B M}_{2}\right]$, where they were labeled (1.4) and (1.5), respectively.

In Diagram I, an s-image denotes the image under an $s$-map, that is, a map with separable fibers. ${ }^{4}$ A $\sigma$-space is a space with a $\sigma$-locally finite network. When implications in the diagram require additional hypotheses, these are indicated next to the relevant arrows. ${ }^{5}$ The notation "all $P \in \mathcal{P}$ closed" next to the implications $(1.3) \rightarrow(1.1)$ and (1.4) $\rightarrow$ (1.6) refers to the collection $\mathscr{P}$ in the definitions of these conditions given above.

Many of the implications in Diagram I follow immediately from the definitions. Some of the others appear in the literature: That $X$ has a point-countable base if and only if it is an open $s$-image of a metric space was proved by Ponomarev [P], and that a regular space $X$ is a quotient image of a separable metric space if and only if it is a $k$-and- $\boldsymbol{\kappa}_{0}$-space was proved in $\left[\mathbf{M}_{1}\right]$; that $(1.2) \rightarrow$ (point-countable base) if $X$ is regular and either $X$ is a $k$-space or $t(X) \leq \omega,{ }^{6}$ as well as the implication $\sigma \rightarrow(1.2)_{p}$, was established in $\left[\mathbf{B M}_{2}\right]$. In this paper, we prove that $X$ is a quotient $s$-image of a metric space if and only if it is a $k$-space satisfying (1.1) in $\S 6$, and that $(1.3) \rightarrow(1.2)$ and $(1.3)_{p} \rightarrow(1.2)_{p}$ in countably bi- $k$-spaces in $\S 3$. In $\S 2$, we prove that $(1.3) \rightarrow(1.4) \stackrel{k}{\rightarrow}(1.3)$, that $(1.3)_{p} \rightarrow(1.4)_{p} \stackrel{k}{\rightarrow}(1.3)_{p}$, that $(1.3) \rightarrow(1.1)$ if all $P \in \mathcal{P}$ are closed, and that $(1.1) \rightarrow(1.5) \stackrel{k}{\rightarrow}(1.1)$. In $\S 5$, we prove that (1.4) $\rightarrow \boldsymbol{\aleph}_{0}$ if $X$ is a regular separable Fréchet space, and

\footnotetext{
${ }^{4} \mathrm{All}$ maps in this paper are continuous and onto.

${ }^{5}$ See Section 3 for the definition of countably bi- $k$-spaces.

${ }^{6} X$ has countable tightness, or $t(X) \leq \omega$, if, whenever $x \in \bar{A}$ in $X$, then $x \in \bar{C}$ for some countable $C \subset A$. Sequential spaces and hereditarily separable spaces have countable tightness.
} 


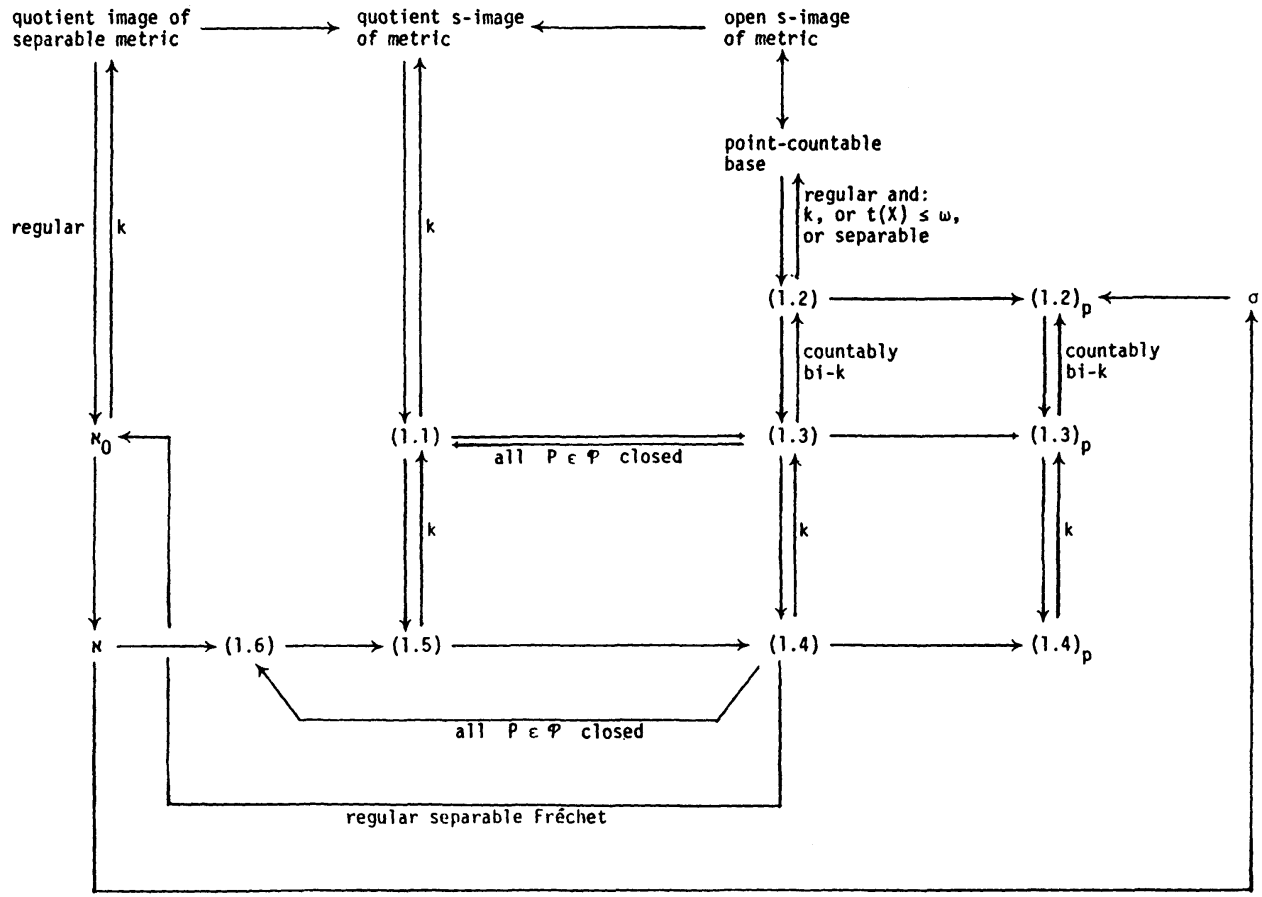

\section{DIAGRAM I}

that (1.2) $\rightarrow$ (point-countable base) if $X$ is regular and separable. The remaining implications in Diagram I are clear.

Besides the results indicated in Diagram I, we also obtain some results concerning hereditarily meta-Lindelöf spaces. In $\S 8$, we prove, among other results, that a pseudo-open ${ }^{7} s$-image of a hereditarily meta-Lindelöf space is hereditarily meta-Lindelöf, and that a regular Fréchet space satisfying (1.3) is hereditarily meta-Lindelöf.

We conclude this introduction by recording some elementary facts which will be used later on.

LEMMA 1.7. If $f: X \rightarrow Y$ is a quotient map, and if $X$ is determined by the cover $\mathcal{P}$, then $Y$ is determined by $f(\mathcal{P})=\{f(P): P \in \mathcal{P}\}$.

LEMMA 1.8. If $\left(X_{\alpha}\right)$ is a cover of $X$, then $X$ is determined by $\left(X_{\alpha}\right)$ if and only if the obvious map $f: \Sigma_{\alpha} X_{\alpha} \rightarrow X$ is quotient (where $\Sigma$ denotes topological sum).

LEMMA 1.9. If $X$ is determined by the cover $\left(X_{\alpha}\right)$, and if each $X_{\alpha}$ is determined by the cover $\mathscr{P}_{\alpha}$, then $X$ is determined by $\cup_{\alpha} \mathscr{P}_{\alpha}$.

${ }^{7} \mathrm{~A}$ map $f: X \rightarrow Y$ is pseudo-open if, for each $y \in Y, y \in(f(U))^{0}$ whenever $U$ is an open subset of $X$ containing $f^{-1}(y)$. 
2. Point-countable $k$-networks and spaces determined by point-countable collections. In this section, we analyze the close relationship between the concept of a point-countable $k$-network (see property (1.4) or, more generally, property $(1.4)_{p}$ ) and spaces determined by $\mathscr{P}$ or $\mathcal{P} *$, where $\mathscr{P}$ is a point-countable collection (see, especially, properties (1.1), (1.3), and $\left.(1.3)_{p}\right)$.

The following is the principal result of this section.

Proposition 2.1. Suppose that $\mathscr{P}$ is a point-countable cover of $X$ such that $X$ is determined by $\mathcal{P} *$. Then every countably compact $K \subset X$ is covered by some finite $\mathscr{F} \subset \mathcal{P}$.

Proof. Suppose not. For each $x \in K$, let $\{P \in \mathscr{P}: x \in P\}=\left\{P_{n}(x)\right.$ : $n \in \omega\}$. Inductively choose $x_{n} \in K$ such that $x_{n} \notin P_{j}\left(x_{i}\right)$ for $i, j<n$. Since $K$ is countably compact, the set $A=\left\{x_{n}: n \in \omega\right\}$ has a cluster point $x^{*}$, so $B=A \backslash\left\{x^{*}\right\}$ is not closed. Thus there exists a finite $\mathscr{F} \subset \mathscr{P}$ such that $B \cap(\cup \mathscr{F})$ is not closed. Some $F \in \mathscr{F}$ must contain infinitely many $x_{n}$ 's. Then $F=P_{j}\left(x_{i}\right)$ for some $i$ and $j$, and there exists $n>i, j$ such that $x_{n} \in P_{j}\left(x_{i}\right)$, contradicting the way the $x_{n}$ 's were chosen.

The following simple result is a partial converse to Proposition 2.1.

LEMMA 2.2. If $\mathscr{P}$ is a cover of a $k$-space $X$ such that every compact $K \subset X$ is covered by some finite $\mathscr{F} \subset \mathcal{P}$, then $X$ is determined by $\mathcal{P} *$.

Proof. The proof is immediate from the following simple observation: If a space $X$ is determined by a cover $\Re$, and $\Re$ is a refinement of $\Re$, then $X$ is determined by $\mathfrak{T}$.

COROLlary 2.3. In any space $X,(1.3) \rightarrow(1.4)$ and $(1.3)_{p} \rightarrow(1.4)_{p}$. The converse implications are true if $X$ is a $k$-space.

Proof. The first assertion follows immediately from Proposition 2.1. The second follows from Lemma 2.2 and the fact that an open subset of a Hausdorff $k$-space is a $k$-space.

Now for another simple lemma.

LEMMA 2.4. If $\mathcal{P}$ is a closed cover of $X$ such that $\mathcal{P}^{*}$ determines $X$, then $\Im$ determines $X$. 
Proof. This follows from a simple observation: If $A \cap P_{i}$ is closed in $P_{i}$ for $i=1,2, \ldots, n$, and if each $P_{i}$ is closed in $X$, then $A \cap P_{i}$ is closed in $X$ for all $i$, and therefore $A \cap\left(\cup_{i=1}^{n} P_{i}\right)$ is also closed in $X$.

COROLlaRY 2.5. If $\mathcal{P}$ satisfies (1.3) and the elements of $\mathcal{P}$ are closed, then $\mathcal{P}$ satisfies (1.1).

There is another simple condition which implies that (1.3) implies (1.1), namely that $X$ is countable (since in this case the collection $\mathscr{P}$, hence also $\mathcal{P}^{*}$, is countable). However, Example 9.7 shows that it does not suffice for $X$ to be merely separable.

For applications of Proposition 2.1 to countably compact spaces, see Section 4.

The following lemma will be used in the proof of Proposition 2.7 and elsewhere in this paper.

Lemma 2.6. Suppose $X$ is determined by a cover $\mathcal{P}$, and $x \in X$. Then:

(a) If $X$ is Fréchet ${ }^{8} x \in(\operatorname{st}(x, \mathcal{P}))^{0}$.

(b) If $X$ is first-countable and $\mathscr{P}$ is point-countable, then there is a finite $\mathscr{F} \subset \mathscr{P}$ such that $x \in \cap \mathscr{F}$ and $x \in(\cup \mathcal{F})^{0}$

Proof. (a) Suppose not. Then there exist $x_{n} \in X \backslash \operatorname{st}(x, \mathcal{P})$ with $x_{n} \rightarrow x$. Then $A=\left\{x_{n}: n \in \omega\right\}$ is not closed, but $A \cap P$ is relatively closed in $P$ for each $P \in \mathcal{P}$, a contradiction.

(b) Suppose not. Let $\left(P_{n}\right)$ enumerate $\{P \in \mathscr{P}: x \in P\}$. Let $\left(U_{n}\right)$ be a countable decreasing base at $x$. Choose $x_{n} \in U_{n} \backslash \cup_{i \leq n} P_{i}$. Then, as in (a), the set $\left\{x_{n}: n \in \omega\right\}$ is not closed, but its intersection with each $P \in \mathscr{P}$ is relatively closed in $P$.

Proposition 2.7. For any space we have $(1.1) \rightarrow(1.5)^{10}$, and $(1.5) \rightarrow$ (1.1) in $k$-spaces.

Proof. Suppose $\mathcal{P}$ is a cover of $X$ satisfying (1.1), and let us show that $\mathscr{P}$ satisfies (1.5). Let $x \in K \subset U$ be as in (1.5). Then $\{P \in \mathscr{P}: P \subset U\}$ determines $U$, so $\{P \cap K: P \in \mathcal{P}, P \subset U\}$ determines $K$. Also, $X$ satisfies

\footnotetext{
${ }^{8}$ Example 9.3 shows that "Fréchet" cannot be weakened to "sequential".

"Example 9.8 shows that "first-countable" cannot be weakened to "Fréchet". It can, however, be weakened to "countably bi-sequential" $\left[\mathbf{M}_{2}\right]$.

${ }^{10}$ It is not known whether (1.1)-or even the existence of a point-countable base-implies (1.6).
} 
(1.4) by Corollary 2.3, hence $K$ also satisfies (1.4), and therefore $K$ is metrizable by Theorem 3.3 in the next section. The conclusion of (1.5) now follows from Lemma 2.6 (with $X$ replaced by $K$, and $\mathcal{P}$ replaced by $\{P \cap K: P \in \mathcal{P}, P \subset U\})$.

Suppose, now, that $X$ is a $k$-space, and that $\mathcal{P}$ is a cover of $X$ satisfying (1.5). To show that $\mathscr{P}$ satisfies (1.1), let $U$ be open in $X$, and suppose that $A \subset U$ is not closed in $U$. We must show that $A \cap P$ is not closed in $P$ for some $P \in \mathcal{P}$ with $P \subset U$. Now $U$, being open in $X$, is a $k$-space, so $A \cap K$ is not closed in $K$ for some compact $K \subset U$. Pick $x \in K \backslash A$ such that $x \in \bar{A}$. By (1.5), there is a $P \in \mathscr{P}$ with $P \subset U$ such that $x \in(A \cap P)^{-}$, and hence $A \cap P$ is not closed in $P$.

3. Point-countable collections in countably bi- $k$-spaces. As defined in $\left[\mathbf{M}_{2}\right], X$ is a countably bi-k-space if, whenever $\left(A_{n}\right)$ is a decreasing sequence of subsets of $X$ with a common cluster point $x$, then there exists a decreasing sequence $\left(B_{n}\right)$ of subsets of $X$ such that $x \in\left(B_{n} \cap A_{n}\right)^{-}$for all $n$, the set $K=\bigcap_{n} B_{n}$ is compact, and each open $U$ containing $K$ contains some $B_{n}$. Locally compact spaces, first countable spaces, and paracompact $M$-spaces ${ }^{11}$ are countably bi- $k$, and every countably bi- $k$ space is a $k$-space. Moreover, all countably bi- $k$-spaces have the following property $\left[\mathbf{M}_{2} ;\right.$ Proposition 4.E.5]:

(3.1) If $\left(A_{n}\right)$ is a decreasing sequence of subsets of $X$, and if $\cap_{n} \bar{A}_{n} \neq \varnothing$, then some compact $K \subset X$ meets every $A_{n}$.

We now state and prove the basic result of this section.

Proposition 3.2. Let $X$ be a countably bi-k-space of countable tightness, ${ }^{12}$ and $\mathscr{P}$ a point-countable cover of $X$ such that each compact $K \subset X$ is covered by some finite $\mathscr{F} \subset \mathscr{P} .{ }^{13}$ Then every $x \in X$ is in $(\cup \mathscr{F})^{0}$ for some finite $\mathscr{F} \subset \mathcal{P}$.

Proof. Suppose the conclusion is false for some $x \in X$. For each countable $C \subset X$, let $\left(P_{i}(C)\right)$ be an enumeration of the countable collection $\{P \in \mathcal{P}: P \cap C \neq \varnothing\}$. Using the countable tightness of $X$, we can inductively choose countable sets $C_{n} \subset X$, with $C_{1}=\{x\}$, such that $x \in \bar{C}_{n}$

\footnotetext{
${ }^{11} X$ is a paracompact $M$-space if it is Hausdorff and admits a perfect map onto a metric space.

${ }^{12}$ The "countably bi- $k$-space" assumption can be weakened to (3.1), but it cannot be weakened to " $k$-space" (see Example 9.2). It is not known whether the assumption that $X$ has countable tightness (see Footnote 6) can be omitted.

${ }^{13}$ Equivalently, by Proposition 2.1 and Lemma 2.2, if $X$ is determined by $\mathcal{P} *$.
} 
for all $n$, and $C_{n} \cap P_{i}\left(C_{j}\right)=\varnothing$ whenever $i<n$ and $j<n$. The last condition implies that no $P \in \mathscr{P}$ meets infinitely many $C_{n}$.

Let $A_{n}=\cup\left\{C_{k}: k \geq n\right\}$. Then $\left(A_{n}\right)$ is decreasing and $x \in \bar{A}_{n}$ for all $n$, so some compact $K \subset X$ meets every $A_{n}$ and hence meets infinitely many $C_{n}$. But $K$ is covered by some finite subcollection $\mathscr{F}$ of $\mathscr{P}$, so some $P \in \mathscr{F}$ meets infinitely many $C_{n}$, a contradiction.

Before applying Proposition 3.2, we pause to quote two results from $\left[\mathbf{B M}_{2}\right]$ which will be frequently used (and generalized) in the sequel.

THEOREM 3.3. [BM $; M_{2}$; Theorem 3.1]. Every compact space satisfying $(1.2)_{p}$ (equivalently, $(1.4)_{p}$ ) is metrizable.

COROLlaRY 3.4. Every $k$-space satisfying $(1.4)_{p}$ is sequential, and thus of countable tightness.

We now come to some consequences of Proposition 3.2 and Corollary 3.4 .

COROLlary 3.5. In any countable bi-k-space, $(1.4) \rightarrow(1.2)$ and $(1.4)_{p}$ $\rightarrow(1.2)_{p}$. (The converses are trivial.)

Proof. We will prove that $(1.4) \rightarrow(1.2)$. The proof that $(1.4)_{p} \rightarrow(1.2)_{p}$ is similar.

Let $U \subset X$ be open. Then $U$ has countable tightness by Corollary 3.4, and $U$ is countably bi- $k$ because it is an open subset of a Hausdorff countably bi- $k$-space [ $\mathbf{M}_{2}$, top of page 114]. Hence, if $\mathcal{P}$ is a point-countable $k$-network for $X$, the hypotheses of Proposition 3.2 are satisfied with $X$ replaced by $U$ and $\mathscr{P}$ by $\{P \in \mathscr{P}: P \subset U\}$. Thus $X$ has property (1.2).

COROllaRY 3.6. If $X$ is regular, then $X$ has a point-countable base if and only if it is countably bi-k and satisfies (1.4).

Proof. The "only if" part is trivial, while the "if" part follows from Corollary 3.5 and the fact, noted in the introduction, that a regular $k$-space satisfying (1.2) has a point-countable base [ $\mathbf{B M}_{2}$, Theorem 6.2].

Corollary 3.5 permits us to sharpen two results from $\left[\mathbf{B M}_{2}\right]$ by weakening (1.2) to $(1.4)_{p}$ in the hypotheses.

COROllaRy 3.7. A space $X$ is metrizable if and only if it is a paracompact $M$-space satisfying $(1.4)_{p}$. 
Proof. With $(1.4)_{p}$ strengthened to $(1.2)_{p}$, this result was proved in $\left[\mathbf{B M}_{2}\right.$; Theorem 4.2]. Since paracompact $M$-spaces are countably bi- $k$, the present result follows from that theorem and Corollary 3.5 .

COROLlaRY 3.8. A regular space is a $\sigma$-space if and only if it is a strong $\sum$-space ${ }^{14}$ satisfying $(1.4)_{p}$.

Proof. This result follows from Corollary 3.7 in essentially the same way that $\left[\mathbf{B M}_{2} ;\right.$ Theorem 5.2] followed from $\left[\mathbf{B M}_{2} ;\right.$ Theorem 4.2], using the easily verified fact that $(1.4)_{p}$ - just like $(1.2)_{p}$-is hereditary and finitely productive. (See $\$ 7$ for the hereditary and productive characteristics of all these properties.)

4. Countably compact spaces. We shall consider conditions (1.4) and $(1.4)_{p}$ with "compact" changed to "countably compact". Let us call these conditions "quasi-(1.4)" and "quasi-(1.4), ", respectively. The relation of these concepts to closely related concepts from Diagram I is shown in the following Diagram II, where only those implications which are valid without additional hypotheses are indicated. The implications (1.3) $\rightarrow$ quasi-(1.4) and $(1.3)_{p} \rightarrow$ quasi-(1.4) $)_{p}$ follow from Proposition 2.1; the others are clear.

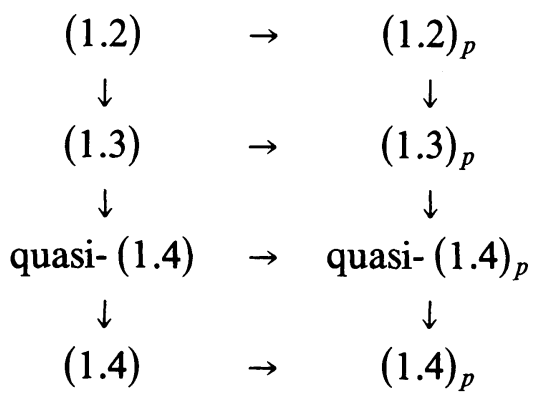

\section{DiAgraM II}

We now quote a result of Balogh [Ba; Theorem 2.1] which generalized the result from $\left[\mathbf{B M}_{2}\right]$ quoted in our Theorem 3.3.

THEOREM 4.1 (Balogh). Every countably compact space satisfying (1.2) (equivalently, quasi-(1.4) $)$ is metrizable (and thus compact).

It may be worth observing that Proposition 2.1 yields a new and direct proof of Theorem 4.1, because Proposition 2.1 implies that the

${ }^{14}$ in the sense of K. Nagami [N]. 
proof of Theorem 3.3 in $\left[\mathbf{B M}_{2}\right.$; Theorem 3.1] remains valid without modification to prove the more general Theorem 4.1. (Proposition 2.1 implies that, even if $X$ is only countably compact, the cover $R$ in the proof of $\left[\mathbf{B M}_{2}\right.$; Theorem 3.1] has a finite subcover.)

It follows from Theorem 4.1 that, in any space satisfying quasi-(1.4) ${ }_{p}$, every countably compact subset is compact, and hence in such a space every property defined in terms of countably compact subsets is equivalent to the analogous property defined in terms of compact subsets. Consequently, the countably compact counterparts of Corollaries 3.5-3.8 are all valid. In particular, we obtain the following analogues of Corollaries 3.7 and 3.8.

COROLlary 4.2. A space $X$ is metrizable if and only if it is an M-space satisfying quasi-(1.4) $)_{p}$.

CoRollary 4.3. A regular space $X$ is a $\sigma$-space if and only if it is a $\Sigma$-space ${ }^{15}$ satisfying quasi-(1.4) . $^{\text {. }}$

5. Separable spaces. The purpose of this section is to examine the effect of separability on properties (1.4) and (1.2).

First we prove the following lemma; part (a) will be applied in the proof of Theorem 5.2, while (b) is used in the proof of Proposition 8.6.

LEMMA 5.1. Suppose $X$ is a regular Fréchet space, $\mathscr{P}$ a $k$-network for $X$, and $x \in U$ with $U$ open in $X$. Then:

(a) If $x \in \bar{A}$ in $X$, then $x \in(P \cap A)^{-}$for some $P \in \mathcal{P}$ such that $\bar{P} \subset U$.

(b) If $B=\cup\{P \in \mathscr{P}: x \in \bar{P} \subset U\}$, then $x \in B^{0}$.

Proof. (a) If $x \in A$, the conclusion is clear. So suppose $x \notin A$. Pick $x_{n} \in A \cap U$ with $x_{n} \rightarrow x$, and let $K=\{x\} \cup\left\{x_{n}: n \in \omega\right\}$. Then $K \subset U$ and $K$ is compact, so $K \subset V \subset \bar{V} \subset U$ for some open $V$ in $X$. Hence $K \subset \cup \mathscr{F} \subset V$ for some finite $\mathscr{F} \subset \mathscr{P}$. Some $P \in \mathscr{F}$ must contain infinitely many $x_{n}$, and this $P$ has the required property.

(b) Suppose not. Let $A=X \backslash B$. Then $x \in \bar{A}$, so, by (a), $x \in(P \cap A)^{-}$ for some $P \in \mathcal{P}$ with $\bar{P} \subset U$. But then $P \subset B$ by definition of $B$, so $P \cap A=\varnothing$, contradicting $x \in(P \cap A)^{-}$.

\footnotetext{
${ }^{15}$ in the sense of K. Nagami [N].
} 
THEOREM 5.2. The following are equivalent for a regular Fréchet space $X:$

(a) $X$ is an $\aleph_{0}$-space.

(b) $X$ is a separable space satisfying (1.4).

Proof. That (a) $\rightarrow$ (b) is trivial. So suppose $X$ satisfies (b). Let $Q$ be a countable dense subset of $X$, and let $\mathcal{P}$ be a point-countable $k$-network for $X$. Let $\Re=\{\bar{P}: P \in \mathcal{P}$ and $P \cap Q \neq \varnothing\}$. Certainly $\Re$ is countable. We will show that $\Re$ is a $k$-network for $X$.

Suppose $K \subset U$, where $K$ is compact and $U$ is open in $X$. Let $\Re(U)=\{R \in \Re: R \subset U\}$. It follows from Lemma 5.1(a) (with $A=Q$ ) that $\Re(U)$ covers $U$. We must show that a finite subcollection of $R(U)$ covers $K$, and we will do that by showing that each $x \in K$ is in the relative interior, in the subspace $K$, of a finite union of elements of $\Re(U)$.

Suppose this is false for some $x \in K$. Let $\mathscr{R}(U)=\left\{R_{n}: n \in \omega\right\}$, with $x \in R_{0}$. Now $K$ is first-countable by Theorem 3.3, so we can choose $x_{n} \in K \backslash \cup_{i \leq n} R_{i}$ such that $x_{n} \rightarrow x$. Since each $R_{n}$ is closed, we can also choose $q_{n, k} \in Q \backslash \cup_{i \leq n} R_{i}$ such that $q_{n, k} \rightarrow x_{n}$ as $k \rightarrow \infty$. But then $x$ is in the closure of these $q_{n, k}$ 's, so there exists a sequence $q_{n_{j}, k_{j}} \rightarrow x$ as $j \rightarrow \infty$. Since $x_{n} \neq x$ and $q_{n, k} \neq x$ for all $n$ and $k$ (because $x \in R_{0}$ ), we have $n_{j} \rightarrow \infty$ as $j \rightarrow \infty$. By Lemma 5.1(a), there exists a $P \in \mathscr{P}$ such that $\bar{P} \subset U$ and $q_{n_{j}, k_{j}} \in P$ for infinitely many $j$. Then $\bar{P} \in \mathscr{R}(U)$, so $\bar{P}=R_{m}$ for some $m \in \omega$. But $q_{n_{j}, k_{j}} \notin R_{m}$ when $n_{j} \geq m$, a contradiction.

Examples 9.3, 9.5 and 9.7 show that, in Theorem 5.2 (b) $\rightarrow$ (a), "Fréchet space" cannot be weakened to "sequential space" (which, by Corollary 3.6, is here equivalent to " $k$-space"). Examples 9.4 and 9.6 show that "separable" cannot be replaced by "Lindelöf". Note that, if "Fréchet" is replaced by "countably bi- $k$ " in the hypothesis of Theorem 5.2, then, by Corollary 3.6, condition (b) (and hence also condition (a)) implies that $X$ is a separable metric space.

We conclude this section with the following result. It should be observed that, if $X$ is a $k$-space, this result follows immediately from $\left[\mathbf{B M}_{2}\right.$, Theorem 6.2]; see Diagram I.

PROPOSITION 5.3. The following are equivalent for a regular space $X$ :

(a) $X$ is a separable metric space.

(b) $X$ is a separable space satisfying (1.2).

Proof. That (a) $\rightarrow$ (b) is trivial. Suppose $X$ satisfies (b). Let $D$ be a countable dense subset of $X$, and let $\mathcal{P}$ be a cover of $X$ satisfying (1.2). Let 
$\Re=\{\bar{P}: P \in \mathscr{P}$ and $P \cap D \neq \varnothing\}$. Then $\Re$ is countable; we will prove that $\Re$ is a network for $X$. Suppose $x \in U$, with $U$ open in $X$. Let $\mathscr{F}$ be a finite subset of $\mathscr{P}$ such that $x \in(\cup \mathscr{F})^{0} \subset \overline{U \mathscr{F}} \subset U$. Then $x \in$ $(\cup\{F \cap D: F \in \mathscr{F}\})^{-}=\bigcup\left\{(F \cap D)^{-}: F \in \mathscr{F}\right\}$. Thus, for some $F \in \mathscr{F}$, we have $x \in(F \cap D)^{-}$. Then $x \in \bar{F} \subset U$, and $F \in R$. Hence $\Re$ is a countable network for $X$.

Every space with a countable network is hereditarily separable and thus has countable tightness. Hence $X$ has a point-countable base by $\left[\mathbf{B M}_{2}\right.$; Theorem 6.2], and so $X$ (being separable) satisfies (a).

6. Quotient s-images of metric spaces. Quotient $s$-images of metric spaces were characterized by $\mathrm{T}$. Hoshina in [H]. In Theorem 6.1 we obtain a new characterization in terms of concepts considered elsewhere in this paper, while simultaneously giving a partial solution to a problem stated in [MN].

Recall that a map $f: X \rightarrow Y$ is compact-covering if every compact $K \subset Y$ is the image of a compact $C \subset X$. Analogously, call $f: X \rightarrow Y$ sequence-covering if every convergent sequence (including its limit) $S \subset Y$ is the image of some compact set (not necessarily a convergent sequence) $C \subset X$. Clearly every compact-covering map is sequence-covering, and, analogously to $\left[\mathbf{M}_{1}\right.$, Lemma 11.2$]$, every sequence-covering map onto a sequential Hausdorff space is quotient. ${ }^{16}$ Examples 9.13 and 9.15 show that neither of these implications can be reversed. It was asked in [MN] whether, nevertheless, every quotient $s$-image $Y$ of a metric space must also be a compact-covering quotient $s$-image of a (possibly different) metric space. While this equation remains open, Theorem 6.1 shows that such a space $Y$ must at least be a sequence-covering quotient $s$-image of a metric space.

THEOREM 6.1. The following are equivalent for a space $X$ :

(a) $X$ is a sequence-covering quotient s-image of a metric space.

(b) $X$ is a quotient s-image of a metric space.

(c) $X$ is a $k$-space satisfying (1.1).

Proof. (a) $\rightarrow$ (b): Trivial.

(b) $\rightarrow$ (c): Let $\mathscr{B}$ be a point-countable base for the metric space $M$, and let $f: M \rightarrow X$ be a quotient $s$-map. Then $X$ is a $k$-space because it is

\footnotetext{
${ }^{16}$ Proof. Let $f: X \rightarrow Y$ be such a map. Suppose $A \subset Y$ with $f^{-1}(A)$ closed in $X$. If $S \subset Y$ is a convergent sequence, then $S=f(K)$ for some compact $K \subset X$. Since $f^{-1}(A) \cap K$ is compact, so is $A \cap S$, and hence $A \cap S$ is closed. Since $Y$ is sequential, $A$ is closed in $Y$.
} 
the quotient image of a $k$-space. We now show that $\mathscr{P}=\{f(B): B \in \mathscr{B}\}$ satisfies the conditions of (1.1). First, $\mathscr{P}$ is point-countable, because each $f^{-1}(x)$ is separable and hence $f^{-1}(x)$ meets only countably many members of $\mathscr{B}$. Let $U \subset X$ be open. Then $f \mid f^{-1}(U)$ is a quotient map of $f^{-1}(U)$ onto $U$, so, by $1.7, U$ is determined by $\left\{f(B): B \in \mathscr{B}\right.$ and $\left.B \subset f^{-1}(U)\right\}=$ $\{P \in \mathscr{P}: P \subset U\}$.

(c) $\rightarrow$ (a). Let $X$ be a $k$-space with a cover $\mathscr{P}$ satisfying (1.1). We may assume that $X \in \mathcal{P}$. Giving $\mathcal{P}$ the discrete topology, the countable product $\rho^{\omega}{ }^{\omega}$ is metrizable. Let $M \subset \mathcal{P}^{\omega}$ be the set of all $\left(P_{n}\right) \in \mathcal{P}^{\omega}$ such that, for some $x \in \bigcap_{n} P_{n}$, every neighborhood of $x$ contains some $P_{n}$. Since $X$ is Hausdorff, this $x$ is uniquely determined by $\left(P_{n}\right)$ (in fact, $\{x\}=\bigcap_{n} P_{n}$ ), and we denote it by $f\left(\left(P_{n}\right)\right)$. That defines our map $f: M \rightarrow X$. We will show that $f$ is a sequence-covering quotient $s$-map from $M$ onto $X$.

$f$ is onto: If $x \in X$, and if $\left(P_{n}\right)$ enumerates $\{P \in \mathcal{P}: x \in P\}$, then $f\left(\left(P_{n}\right)\right)=x$.

$f$ is continuous: Clear.

$f$ is an s-map: Clear, since $f^{-1}(x) \subset\{P \in \mathcal{P}: x \in P\}^{\omega}$, and $\mathscr{P}$ is point-countable.

$f$ is sequence-covering: Let $S$ be a convergent sequence in $X$, together with its limit point $s$. We must find a compact $K \subset M$ with $f(K)=S$.

Let $\mathcal{P}^{\prime}$ be the collection of all $P \in \mathscr{P}$ such that $P \cap S$ is a non-empty closed subset of $S$. (Note that $P \cap S$ is closed if either $P \cap S$ is finite or $s \in P$.) Since $S$ is countable, so is $\mathscr{P}^{\prime}$. Let $\left(\mathscr{F}_{n}\right)$ enumerate the finite subcollections of $\mathscr{P}^{\prime}$ which cover $S$. Let $K$ be the set of all $\left(P_{n}\right) \in \Pi_{n} \mathscr{F}_{n}$ such that $\left(P_{n} \cap S\right)$ has the finite intersection property. Now $\Pi_{n} \mathscr{F}_{n}$ is a compact subset of $\mathscr{P}^{\omega}$ (since it is a product of finite spaces), and $K$ is closed in $\Pi_{n} \mathscr{F}_{n}$, so $K$ is a compact subset of $\mathcal{P}^{\omega}$. We will show that $K \subset M$ and $f(K)=S$.

First we show that $K \subset M$ and $f(K) \subset S$. Suppose $\left(P_{n}\right) \in K$. Then $\left(P_{n} \cap S\right)$ is a collection of closed sets with the finite intersection property, so there is an $x \in \bigcap_{n}\left(P_{n} \cap S\right)$. To establish that $\left(P_{n}\right) \in M$ and $f\left(\left(P_{n}\right)\right)=$ $x$, it will suffice to show that every neighborhood $U$ of $x$ contains some $P_{n}$.

For each $y \in S$, pick a neighborhood $W_{y}$ of $y$ in $X$ such that $W_{y} \subset U$ if $y=x$, such that $x \notin W_{y}$ if $y \neq x$, and such that $W_{y} \cap S=\{y\}$ if $y \neq s$. Now $X$ satisfies (1.5) by Proposition 2.7, so there is a finite $\mathscr{C} \subset \mathcal{P}$ such that $s \in \cap \mathbb{Q}, \cup \mathbb{Q} \subset W_{s}$, and $\mathbb{Q}$ covers a neighborhood of $s$ in $S$. Let $T=S \backslash \cup \mathcal{Q}$; clearly $T$ is finite. For each $y \in T$, pick $P_{y} \in \mathcal{P}$ such that $y \in P_{y} \subset W_{y}$, and let $\mathscr{F}=\mathscr{Q} \cup\left\{P_{y}: y \in T\right\}$. Then $\mathscr{F} \subset \mathscr{P}^{\prime}, \mathscr{F}$ covers $S$, and $\mathscr{F}$ is finite, so $\mathscr{F}=\mathscr{F}_{n}$ for some $n$, and hence $P_{n} \in \mathscr{F}$ for this $n$. But our construction of $\mathscr{F}$ implies that $P \subset U$ whenever $x \in P \in \mathscr{F}$, and therefore $P_{n} \subset U$. 
It remains to show that $S \subset f(K)$. Suppose $x \in S$. For each $n$, pick $P_{n} \in \mathscr{F}_{n}$ so that $x \in P_{n}$. Then $\left(P_{n}\right) \in K$, so $\left(P_{n}\right) \in M$. Since $x \in \bigcap_{n} P_{n}$, and since $f\left(\left(P_{n}\right)\right)$ is the only element of $\bigcap_{n} P_{n}$, it follows that $f\left(\left(P_{n}\right)\right)=x$.

$f$ is quotient: Since $X$ is a $k$-space satisfying (1.1), it is sequential by Corollary 3.4. Hence $f$ is a sequence-covering map onto a sequential space, and such maps are always quotient (see Footnote 16).

REMARK. As the proof of Theorem 6.1, (c) $\rightarrow$ (a), shows, any space (not necessarily a $k$-space) satisfying (1.1) - or even (1.5) - is a sequencecovering $s$-image of a metric space.

REMARK. Each of the following two conditions (d) and (e) implies the equivalent conditions (a)-(c) of Theorem 6.1, and (d) $\rightarrow$ (e) by $\left[\mathbf{M}_{5}\right.$, Theorem 3.2]. We don't know whether, conversely, $((\mathrm{a})-(\mathrm{c})) \rightarrow(\mathrm{e})$ or (e) $\rightarrow$ (d). (See Footnote 10.)

(d) $X$ is a $k$-space satisfying (1.6).

(e) $X$ is a compact-covering quotient $s$-image of a metric space.

Recall that if $f: X \rightarrow Y$ is a quotient map and $Y$ is a Fréchet space, then $f$ must be pseudo-open (see Footnote 7), and that the pseudo-open image of a Fréchet space is Fréchet [A]. Thus we have the following corollary to Theorem 6.1.

COROLlaRY 6.2. The following are equivalent for a space $X$ :

(a) $X$ is a pseudo-open s-image of a metric space.

(b) $X$ is a Fréchet space satisfying (1.1).

We don't know whether the class of spaces characterized by Corollary 6.2 is preserved by pseudo-open $s$-images (or even perfect images). See Theorem $7.1(\mathrm{e})-(\mathrm{h})$ for some related results.

Example 9.8 shows that, in general, the spaces characterized by Theorem 6.1 are not preserved by quotient $s$-images or even perfect $s$-images. We do, however, have the following partial result.

Proposition 6.3. Suppose that $X$ is a $k$-space satisfying (1.1) and that $f: X \rightarrow Y$ is a quotient s-map. Then either (a) or (b) implies that $Y$ is a $k$-space satisfying (1.1).

(a) $X$ has a point-countable base.

(b) $X$ is a $k$-and-א-space. 
Proof. Part (a) is the same as the proof of Theorem 6.1, (b) $\rightarrow$ (c). Part (b) is quite similar: Clearly $Y$ is again a $k$-space. Let $\mathcal{P}$ be a $\sigma$-locally-finite $k$-network for $X$. Then, as in the proof of Theorem $6.1,(\mathrm{~b}) \rightarrow(\mathrm{c})$, the collection $2=\{f(P): P \in \mathscr{P}\}$ satisfies (1.1) for $Y$ provided it is point-countable. Now 2 is point-countable provided each $f^{-1}(y)$ meets only countably many members of $\mathscr{P}$. This will be the case if each $f^{-1}(y)$ is Lindelöf. Foged [Fo] has shown that every $k$-and- $\boldsymbol{\kappa}$-space is meta-Lindelöf (i.e., each open cover has a point-countable open refinement). But separable meta-Lindelöf spaces are Lindelöf, so each $f^{-1}(y)$ is Lindelöf.

As the proof shows, Proposition 6.3(b) remains valid with "quotient $s$-map" changed to "quotient map with Lindelöf fibers". That is not true, however, for Proposition 6.3(a), because Example 9.6 shows that even a quotient image of a Lindelöf space with a point-countable base need not satisfy (1.1).

The following result generalizes [ $\mathbf{T}_{\mathbf{1}}$; Theorem 7], where it is shown that (a) and (c) are equivalent if $X$ is metrizable.

Proposition 6.4. Let $f: X \rightarrow Y$ be a closed mapping of a paracompact $\aleph$-space $X$ onto a $k$-space $Y$. Then the following are equivalent:

(a) $Y$ satisfies (1.6).

(b) $Y$ satisfies (1.1).

(c) $\partial f^{-1}(y)$ is separable (equivalently, Lindelöf) for each $y \in Y$.

Proof. By Diagram I, we have (a) $\rightarrow$ (b). Now assume (b), and suppose (c) does not hold. Then some $\partial f^{-1}(y)$ is not Lindelöf, so, by the paracompactness of $X$, there exists a closed discrete subset of $\partial f^{-1}(y)$ of cardinality $\omega_{1}$. The space $Y$ is sequential by Corollary 3.4. Then, since $X$ is paracompact, it follows from [ $\mathbf{T}_{2}$; Lemma 1.5] that $Y$ contains a closed copy of $S_{\omega_{1}}$, the space obtained by identifying the limit points of $\omega_{1}$ convergent sequences. In Example 9.2, we show that this space does not satisfy (1.1). Since it is easily seen that property (1.1) is hereditary with respect to closed subspaces, this contradicts (b). Thus (b) $\rightarrow(c)$.

It remains to prove (c) $\rightarrow$ (a). Assume that each $\partial f^{-1}(y)$ is Lindelöf. For $y \in Y$, choose a point $x(y) \in f^{-1}(y)$. Let $X^{\prime}=\bigcup\left\{\partial f^{-1}(y): y \in Y\right\}$ $\cup\{x(y): y$ is an isolated point of $Y\}$. Then $X^{\prime}$ is a closed subspace of $X$. Let $g$ be the restriction of $f$ to $X^{\prime}$. Then $g$ is a closed map of $X^{\prime}$ onto $Y$, and each $g^{-1}(y)$ is Lindelöf.

Let $\mathscr{P}$ be a $\sigma$-locally-finite closed $k$-network for $X^{\prime}$, and let $\mathcal{Q}=g(\mathscr{P})$. Clearly 2 is a point-countable, closed cover of $Y$. To complete the proof, we show that 2 is a $k$-network for $Y$. 
Suppose $K \subset U \subset Y$, where $K$ is compact and $U$ is open. By $\left[\mathbf{M}_{4}\right], g$ is compact-covering, so there is a compact set $L \subset X$ such that $g(L)=K$. Let $\mathscr{F}$ be a finite subcollection of $\mathcal{P}$ such that $L \subset \cup \mathcal{F} \subset g^{-1}(U)$. Then $K \subset \cup\{g(P): P \in \mathscr{F}\} \subset U$, so 2 is a point-countable closed $k$-network for $Y$.

\section{Preservation.}

THEOREM 7.1. The properties in Diagrams I and II are preserved under the following operations.

(a) All subsets: (1.2), (1.2) $,(1.4),(1.4)_{p}$, quasi-(1.4), quasi-(1.4),$(1.5)$,

(b) All closed subsets, all open subsets, and all subspaces which are k-spaces: (1.1), (1.3), (1.3), .

(c) Countable products: (1.2), (1.2) $,(1.4),(1.4)_{p}$.

(d) Products with locally compact metric spaces: (1.1), (1.3).

(e) Perfect maps: (1.2), (1.2), (1.4), (1.4) .

(f) Open s-maps: Fréchet spaces satisfying (1.1).

(g) Quotient s-maps with Fréchet domain: (1.3), (1.4).

(h) Quotient maps with countable fibers: (1.1), (1.3).

(i) Shrinking closed subsets to a point: $(1.3),(1.3)_{p}$.

Proof of (a). Suppose that $Y \subset X$, and that $\mathscr{P}$ is a cover of $X$ satisfying one of the conditions in (a). Then it is easy to check that $\{P \cap Y: P \in \mathcal{P}\}$ satisfies the same condition for $Y$.

Proof of (b). That (1.1), (1.3) and (1.3) $)_{p}$ are hereditary with respect to closed subsets or open subsets is easy to see.

Suppose $Y$ is a $k$-subspace of $X$ satisfying (1.3). Then $X$ satisfies (1.4) by Corollary 2.3, hence $Y$ satisfies (1.4) by (a) above, and hence $Y$ satisfies (1.3) by Corollary 2.3. Thus (1.3) is preserved by $k$-subspaces, and the same proof works for (1.3) $)_{p}$. The proofs for (1.4) and (1.4) $)_{p}$ are similar, relying on Proposition 2.7 instead of Corollary 2.3.

Proof of (c). Let $X=\Pi_{n} X_{n}$ be a countable product. For each $n \in \omega$, let $\mathscr{P}_{n}$ be a cover of the space $X_{n}$, and let

$$
\mathscr{P}=\left\{P_{0} \times P_{1} \times \cdots \times P_{n} \times X_{n+1} \times X_{n+2} \times \cdots: n \in \omega, P_{i} \in \mathscr{P}_{i}\right\} .
$$

If each $\mathscr{P}_{n}$ is point-countable, so is $\mathscr{P}$. We claim that, if each $\mathscr{P}_{n}$ satisfies $(1.2),(1.2)_{p},(1.4)$, or $(1.4)_{p}$, so does $\mathscr{P}$. The easy verification for (1.2) and $(1.2)_{p}$ is left to the reader, and the proof for $(1.4)_{p}$ is similar to that for (1.4). It remains to give a proof for (1.4). 
Suppose each $\mathscr{P}_{n}$ satisfies (1.4). To show that $\mathscr{P}$ satisfies (1.4), let $K \subset U$ with $K$ compact and $U$ open in $X$. Pick basic open sets $V(1), \ldots, V(m)$ in the product space $X$ such that $K \subset \cup_{i=1}^{m} V(i) \subset U$. Pick a closed cover $A(1), \ldots, A(m)$ of $K$ such that $A(i) \subset V(i)$ for $i=1, \ldots, m$. It is easy to see that, for each $i \leq m$, there is a finite $\mathcal{P}(i) \subset \mathcal{P}$ such that $A(i) \subset \cup \mathscr{P}(i) \subset V(i)$. Let $\mathscr{F}=\bigcup_{i=1}^{m} \mathscr{P}(i)$. Then $\mathscr{F}$ is a finite subcollection of $\mathscr{P}$, and $K \subset \cup \mathscr{F} \subset U$. Hence $\mathscr{P}$ satisfies (1.4).

Proof of (d). First we establish the following fact: If $X$ is determined by $\mathcal{P}$, and $Y$ is locally compact, then $X \times Y$ is determined by $\{P \times Y$ : $P \in \mathscr{P}\}$. To see this, let $E=\Sigma \mathscr{P}$, the topological sum of the elements of $\mathcal{P}$, and let $f: E \rightarrow X$ be the obvious map. By Lemma $1.8, f$ is a quotient map. By Whitehead's theorem [W; Lemma 4], $f \times$ id $_{Y}: E \times Y \rightarrow X \times Y$ is also a quotient map. Since $E \times Y=\Sigma\{P \times Y: P \in \mathcal{P}\}$, the conclusion follows from Lemma 1.8 .

We now complete the proof of (d) for (1.3); the proof for (1.1) is similar. Suppose $\mathscr{P}$ satisfies (1.3) for $X$. Let $Y$ be a locally compact metric space, and let $\mathscr{B}$ be a point-countable base for $Y$. Then $\{P \times B: P \in \mathscr{P}$, $B \in \mathscr{B}\}$ is a point-countable cover of $X \times Y$. It suffices to show that every basic open set $U \times B$ in $X \times Y$, with $U$ open in $X$ and $B \in \Re$, is determined by $\{P \times B: P \in \mathcal{P}, P \subset U\}^{*}=\{P \times B: P \in \mathcal{P} *, P \subset U\}$. But this follows from the previous paragraph and the assumption that $U$ is determined by $\{P \in \mathcal{P} *: P \subset U\}$.

Proof of (e). For (1.2) $)_{p}$, this was proved in Theorem 8.4 of $\left[\mathbf{B M}_{2}\right]$. The proofs in the other cases are similar, and are thus omitted.

Proof of (f). Suppose $f: X \rightarrow Y$ is an open $s$-map, and $X$ is a Fréchet space satisfying (1.1). For each $y \in Y$, let $D_{y}$ be a countable dense subset of $f^{-1}(y)$, and let $D=\cup_{y \in Y} D_{y}$. Then $D$ satisfies (1.1), by part (b) of this theorem. It is easily checked that $f \mid D$ is open. Since $f \mid D$ is countable-toone, the result now follows from part $(\mathrm{h})$.

Proof of (g). Suppose that $f: X \rightarrow Y$ is a quotient $s$-map with $X$ Fréchet, and that $\mathscr{P}$ is a cover of $X$ satisfying (1.4). Let $D=\cup_{y \in Y} D_{y}$ be as in the proof of $(\mathrm{f})$, and let $\mathcal{Q}=\{f(P \cap D): P \in \mathcal{P}\}$. We will show that 2 satisfies (1.3) for $Y$. Since (1.3) implies (1.4), that will prove our result for both (1.3) and (1.4).

Clearly 2 is point-countable. Suppose $V \subset Y$ is open in $Y$. We must show that, if $B \subset V$ and $B$ is not closed in $V$, then $B \cap E$ is not closed in $E$ for some $E \in\{Q \in \mathcal{Q}: Q \subset V\}^{*}$. 
Let $A=f^{-1}(B)$ and $U=f^{-1}(V)$. Since $f$ is quotient, $A$ is not closed in $U$. Pick $x \in U \backslash A$ with $x \in \bar{A}$. Then $x \in(A \cap D)^{-}$, so there are $x_{n} \in A \cap D$ with $x_{n} \rightarrow x$. Let $K=\{x\} \cup\left\{x_{n}: n \in \omega\right\}$. Then $K$ is compact and $K \subset U$, so $K \subset \cup \mathscr{F} \subset U$ for some finite $\mathscr{F} \subset \mathscr{P}$; we may assume that some $P \in \mathscr{F}$ intersects $D_{f(x)}$. Let $E=\bigcup\{f(P \cap D): P \in \mathscr{F}\}$. Then $E \in\{Q \in \mathcal{Q}: Q \subset V\}^{*}$, and $B \cap E$ is not closed in $E$ because $f(x) \in$ $E \backslash B$ and $f(x) \in \bar{B}$.

Proof of (h). This follows easily from Lemma 1.7 and the fact that the image of a point-countable collection under a countable-to-one map is point-countable.

Proof of (i). We will prove this for (1.3). Suppose $X$ has a cover $\mathscr{P}$ satisfying the requirements of (1.3), let $A \subset X$ be closed, let $Y=X / A$, and let $f: X \rightarrow Y$ be the quotient map. Let

$$
\mathcal{Q}=\{f(A)\} \cup\{f(P \backslash A): P \in \mathcal{P}\} .
$$

It is easy to check that the cover 2 of $Y$ satisfies the requirements of (1.3).

Examples 9.10 and 9.11 show that (1.1) and (1.3) are not preserved by arbitrary subsets or binary products. ${ }^{17}$ Example 9.16 shows that (1.4) is not preserved by the maps considered in Theorem 7.1(f)-(h). See $\$ 10$ for some related open problems.

8. Hereditarily meta-Lindelöf spaces. Recall that a space $X$ is meta-Lindelöf if every open cover of $X$ has a point-countable open refinement. The primary purpose of this section is to study hereditarily meta-Lindelöf spaces (i.e., spaces all of whose subspaces are meta-Lindelöf) and their relationship to some of the other concepts considered in this paper.

We begin with a useful characterization. By a well-ordered cover $\left(U_{\alpha}\right)_{\alpha \in A}$ of $X$, we will simply mean a cover with a well-ordered index set $A$; the order need not be related to subset ordering. If $\left(U_{\alpha}\right)$ is such a cover, and if $x \in X$, then $\alpha(x)$ will denote the first $\alpha$ such that $x \in U_{\alpha}$, and $\tilde{U}_{\alpha}$ will denote $\{x \in X: \alpha(x)=\alpha\}$. Clearly $\tilde{U}_{\alpha} \subset U_{\alpha}$.

THEOREM 8.1. ${ }^{18}$ The following are equivalent:

(a) $X$ is hereditarily meta-Lindelöf.

\footnotetext{
${ }^{17}$ We don't know how (1.3) behaves in these respects.

${ }^{18}$ This result was also noticed by J. Chaber and $\mathrm{H}$. Junnila (personal communication).
} 
(b) Every well-ordered open cover $\left(U_{\alpha}\right)$ of $X$ has a point-countable open refinement $\mathcal{W}$ such that, if $x \in X$, then $x \in V \subset U_{\alpha(x)}$ for some $V \in \mathcal{V}$.

(c) Every well-ordered open cover $\left(U_{\alpha}\right)$ of $X$ has a point-countable open refinement $\left(V_{\alpha}\right)$ such that $\tilde{U}_{\alpha} \subset V_{\alpha} \subset U_{\alpha}$ for all $\alpha$.

Proof. (a) $\rightarrow$ (b). Assume this is false. Then we can find a hereditarily meta-Lindelöf space $X$, an ordinal $\lambda$, and a well-ordered open cover $\left(U_{\alpha}\right)_{\alpha<\lambda}$ of $X$ which does not have a refinement $\mathfrak{V}$ satisfying the conditions of (b). We can assume that $\lambda$ is the least such ordinal (for any hereditarily meta-Lindelöf space). Then, for each $\alpha<\lambda$, the well-ordered open cover $\left(U_{\beta}\right)_{\beta<\alpha}$ of $\cup_{\beta<\alpha} U_{\beta}$ has a point-countable open refinement $\mathfrak{V}_{\alpha}$ satisfying the conditions of (b). If $\lambda=\alpha+1$ for some $\alpha$, then it is easy to see that $\mathfrak{V}=\mathfrak{V}_{\alpha} \cup\left\{U_{\alpha}\right\}$ is a refinement of $\left(U_{\alpha}\right)_{\alpha<\lambda}$ satisfying the conditions of (b), a contradiction. So suppose $\lambda$ is a limit ordinal.

Let $\psi$ be a point-countable open refinement of the open cover $\left(U_{\alpha}\right)_{\alpha<\lambda}$ of $X$. For each $W \in \mathscr{W}$, pick $\alpha(W)<\lambda$ such that $W \subset U_{\alpha(W)}$. Now define

$$
\mathscr{V}=\left\{W \cap V: W \in \mathscr{W}, V \in \mathfrak{V}_{\alpha(W)+1}\right\}
$$

Since $\mathscr{W}$ and all $\mathcal{V}_{\alpha}$ are point-countable open collections, so is $\mathcal{T}$. Suppose $x \in X$, and pick $W \in \mathcal{W}$ with $x \in W$. Then

$$
x \in U_{\alpha(W)} \subset \bigcup\left\{U_{\beta}: \beta<\alpha(W)+1\right\},
$$

so there exists $V \in \mathfrak{V}_{\alpha(W)+1}$ with $x \in V \subset U_{\alpha(x)}$. Hence $W \cap V \in \mathfrak{V}$, and $x \in W \cap V \subset U_{\alpha(x)}$. Thus $\mathcal{V}$ is a refinement of $\left(U_{\alpha}\right)_{\alpha<\lambda}$ satisfying the conditions of $(b)$, and we again have a contradiction.

(b) $\rightarrow$ (c). Let $\left(U_{\alpha}\right)$ be a well-ordered open cover of $X$, and choose an open cover $\mathcal{V}$ of $X$ as in (b). For each $\alpha$, let

$$
V_{\alpha}=\bigcup\left\{V \in \mathcal{V}: V \subset U_{\alpha}, V \not \subset U_{\beta} \text { if } \beta<\alpha\right\} .
$$

It is easy to verify that $\left(V_{\alpha}\right)$ satisfies (c).

(c) $\rightarrow$ (a). Suppose $Y \subset X$, and $X$ satisfies (c). Let $\mathscr{W}=\left(W_{\alpha}\right)_{\alpha<\lambda}$ be a well-ordered open cover of $Y$. Let $\left(U_{\alpha}\right)_{\alpha \leq \lambda}$ be a well-ordered open cover of $X$ such that $U_{\lambda}=X$ and, for each $\alpha<\lambda, U_{\alpha} \cap Y=W_{\alpha}$. Let $\left(V_{\alpha}\right)_{\alpha \leq \lambda}$ be a point-countable open refinement of $\left(U_{\alpha}\right)_{\alpha \leq \lambda}$ with $\tilde{U}_{\alpha} \subset V_{\alpha} \subset U_{\alpha}$ for all $\alpha$. Note that each $y \in Y$ is in $\tilde{U}_{\alpha}$ for some $\alpha<\lambda$. It follows that $\left(V_{\alpha} \cap Y\right)_{\alpha<\lambda}$ is a point-countable open refinement of $\mathscr{W}$. Hence $Y$ is meta-Lindelöf, and so $X$ is hereditarily meta-Lindelöf.

Hereditarily meta-Lindelöf spaces are clearly preserved by subsets and topological sums. The following result is less obvious. 
Proposition 8.2. If $f: X \rightarrow Y$ is a pseudo-open ${ }^{19}$ s-map or a closed map, ${ }^{20}$ and if $X$ is hereditarily meta-Lindelöf, then so is $Y$.

Proof. By Theorem 8.1, it will suffice to show that $Y$ satisfies 8.1(c). So let $\left(U_{\alpha}\right)$ be a well-ordered open cover of $Y$, and let us find the cover $\left(V_{\alpha}\right)$ of $Y$ required by $8.1(\mathrm{c})$.

Let $U_{\alpha}^{\prime}=f^{-1}\left(U_{\alpha}\right)$. Then $\left(U_{\alpha}^{\prime}\right)$ is a well-ordered open cover of $X$, so, by Theorem 8.1, it has a point-countable open refinement $\left(V_{\alpha}^{\prime}\right)$ such that $\left(U_{\alpha}^{\prime}\right)^{\tilde{C}} \subset V_{\alpha}^{\prime} \subset U_{\alpha}^{\prime}$ for all $\alpha$. Observe that $f\left(V_{\alpha}^{\prime}\right) \subset U_{\alpha}$ and $f^{-1}\left(\tilde{U}_{\alpha}\right) \subset\left(U_{\alpha}^{\prime} \tilde{)} \subset\right.$ $V_{\alpha}^{\prime}$ for all $\alpha$.

If $f$ is a pseudo-open $s$-map, let $V_{\alpha}=\left(f\left(V_{\alpha}^{\prime}\right)\right)^{0}$. Clearly $V_{\alpha}$ is open, and $V_{\alpha} \subset f\left(V_{\alpha}^{\prime}\right) \subset U_{\alpha}$. Also $\left(V_{\alpha}\right)$ is point-countable because $f$ is an $s$-map and $\left(V_{\alpha}^{\prime}\right)$ is a point-countable collection of open subsets of $X$, and $\tilde{U}_{\alpha} \subset V_{\alpha}$ because $f^{-1}\left(\tilde{U}_{\alpha}\right) \subset V_{\alpha}^{\prime}$ and $f$ is pseudo-open. Hence $\left(V_{\alpha}\right)$ satisfies 8.1(c).

If $f$ is a closed map, let $V_{\alpha}=\left\{y \in Y: f^{-1}(y) \subset V_{\alpha}^{\prime}\right\}$. Then $V_{\alpha}$ is open because $f$ is closed, and $V_{\alpha} \subset f\left(V_{\alpha}^{\prime}\right) \subset U_{\alpha}$. Moreover, $\left(V_{\alpha}\right)$ is point-countable because $\left(V_{\alpha}^{\prime}\right)$ is point-countable, and $\tilde{U}_{\alpha} \subset V_{\alpha}$ because $f^{-1}\left(\tilde{U}_{\alpha}\right) \subset V_{\alpha}^{\prime}$. Hence $\left(V_{\alpha}\right)$ satisfies 8.1(c).

We don't know whether there is an analogue of Theorem 8.1 for (not hereditarily) meta-Lindelöf spaces, or whether Proposition 8.2 remains valid with "hereditarily" omitted. We do, however, have the following two results in case the index set is $\omega_{1}$.

LEMMA 8.3. The following properties of an open cover $\left(U_{\alpha}\right)_{\alpha<\omega_{1}}$ are equivalent:

(a) $\left(U_{\alpha}\right)$ has a point-countable open refinement.

(b) $\left(U_{\alpha}\right)$ has a point-countable open refinement $\mathscr{V}$ such that, if $x \in X$, then $x \in V \subset U_{\alpha(x)}$ for some $V \in \mathcal{V}$.

(c) $\left(U_{\alpha}\right)$ has a point-countable open refinement $\left(V_{\alpha}\right)$ such that $\tilde{U}_{\alpha} \subset V_{\alpha}$ $\subset U_{\alpha}$ for all $\alpha<\omega_{1}$.

Proof. (a) $\rightarrow$ (b). Let $\mathcal{W}$ be a point-countable open refinement of $\left(U_{\alpha}\right)$. For each $W \in \mathscr{W}$, pick $\alpha(W)<\omega_{1}$ such that $W \subset U_{\alpha(W)}$. Let

$$
\mathfrak{V}=\left\{W \cap U_{\alpha}: W \in \mathscr{W}, \alpha \leq \alpha(W)\right\}
$$

\footnotetext{
${ }^{19}$ See Footnote 7 for the definition of pseudo-open.

${ }^{20}$ That Theorem 8.1 can be used to show preservation of hereditarily meta-Lindelöf spaces under closed maps was pointed out to the first author by J. Chaber.
} 
It is easy to check that this $\mathcal{W}$ has the properties required by (b).

(b) $\rightarrow$ (c). This is proved just like Theorem 8.1(b) $\rightarrow$ (c).

(c) $\rightarrow$ (a). Clear.

Proposition 8.4. If $X$ is meta-Lindelöf, and if every open cover of $X$ has a subcover of cardinality $\leq \boldsymbol{\aleph}_{1}$, then every pseudo-open s-image and every closed image ${ }^{21}$ of $X$ has the same properties.

Proof. Let $X$ satisfy the conditions of the proposition, and suppose $f: X \rightarrow Y$ is a pseudo-open $s$-map or a closed map. It is routine to verify that every open cover of $Y$ has a subcover of cardinality $\leq \boldsymbol{\aleph}_{1}$. To show that $Y$ is meta-Lindelöf, let $\mathscr{Q}$ be an open cover of $Y$. Then $\mathscr{Q}$ has a subcover $\left(U_{\alpha}\right)_{\alpha<\omega_{1}}$. The fact that $\left(U_{\alpha}\right)_{\alpha<\omega_{1}}$ has a point-countable open refinement now follows from Lemma 8.3 just as Proposition 8.2 followed from Theorem 8.1.

Which of the properties considered in Diagram I are hereditarily meta-Lindelöf? Clearly spaces with a point-countable base are. Some stronger results will be presented in Proposition 8.6 below, but first we need the following lemma, which deals with conditions related to 8.1(b).

LEMMA 8.5. Among the following properties of $X,(\mathrm{a}) \leftrightarrow(\mathrm{b})$ and $(\mathrm{c}) \rightarrow(\mathrm{a})$; for regular $X$, all are equivalent.

(a) $X$ is hereditarily meta-Lindelöf.

(b) Every well-ordered open cover $\left(U_{\alpha}\right)$ of $X$ has a point-countable refinement $\mathcal{P}$ such that, if $x \in X$, then

$$
x \in\left(\bigcup\left\{P \in \mathcal{P}: x \in P \subset U_{\alpha(x)}\right\}\right)^{0} .
$$

(c) Same as (b), but with the last formula changed to

$$
x \in\left(\bigcup\left\{P \in \mathscr{P}: x \in \bar{P} \subset U_{\alpha(x)}\right\}\right)^{0} .
$$

Proof. (a) $\rightarrow$ (b). This follows from Theorem 8.1, (a) $\rightarrow$ (b).

(b) $\rightarrow$ (a). It will suffice to show that (b) implies $8.1(\mathrm{c})$. So let $\left(U_{\alpha}\right)$ be a well-ordered open cover of $X$, and choose a cover $\mathscr{P}$ of $X$ satisfying (b). For each $\alpha$, let

$$
V_{\alpha}=\left(\bigcup\left\{P \in \mathscr{P}: P \subset U_{\alpha}, P \not \subset U_{\beta} \text { if } \beta<\alpha\right\}\right)^{0} .
$$

\footnotetext{
${ }^{21}$ For closed images, this result was obtained independently by D. Burke.
} 
As in the proof of $8.1(\mathrm{~b}) \rightarrow 8.1(\mathrm{c})$, it is easy to verify that $\left(V_{\alpha}\right)$ satisfies the requirements of $8.1(\mathrm{c})$.

(c) $\rightarrow$ (a). The proof is essentially the same as (b) $\rightarrow$ (a), but with

$$
V_{\alpha}=\left(\bigcup\left\{P \in \mathcal{P}: \bar{P} \subset U_{\alpha}, \bar{P} \not \subset U_{\beta} \text { if } \beta<\alpha\right\}\right)^{0} .
$$

We omit the straightforward proof that (a) $\rightarrow$ (c) for regular $X$.

Proposition 8.6. Each of the following conditions implies that $X$ is hereditarily meta-Lindelöf.

(a) $X$ is a Fréchet space satisfying (1.1).

(b) $X$ is a regular Fréchet space satisfying (1.3).

Proof. (a). As noted in Corollary 6.2, the Fréchet spaces satisfying (1.1) characterize the pseudo-open $s$-images of metric spaces. Hence these spaces are hereditarily meta-Lindelöf by Proposition 8.2. (Alternatively, this result follows from the implication $8.5(\mathrm{~b}) \rightarrow 8.5(\mathrm{a})$, together with Lemma 2.6 with $X$ replaced by $U_{\alpha(x)}$.)

(b) This follows from the implication $8.5(\mathrm{c}) \rightarrow 8.5(\mathrm{a})$ and from Lemma 5.1(b).

REMARK. It follows from Example 9.3 and Theorem 6.1 that "Fréchet" cannot be weakened to "sequential" in Proposition 8.6, even if $X$ satisfies (1.6). If $X$ is an $\boldsymbol{\aleph}$-space, however, then $X$ is hereditarily meta-Lindelöf whenever it is a $k$-space [Fo].

Our final results in this section deal with locally separable spaces.

Proposition 8.7. Suppose $X$ is locally separable and hereditarily meta-Lindelöf. Then $X$, as well as every pseudo-open s-image of $X$, is a topological sum of separable, hereditarily Lindelöf spaces.

Proof. Let $X$ be locally separable and hereditarily meta-Lindelöf, and let $f: X \rightarrow Y$ be a pseudo-open $s$-map. Each $f^{-1}(y)$, being separable and meta-Lindelöf, is Lindelöf. Since $X$ is locally separable, it then follows that each $f^{-1}(y)$ is contained in some separable open set $U_{y}$. Since $y \in\left(f\left(U_{y}\right)\right)^{0}$, we see that $Y$ is locally separable. Also, $Y$ is hereditarily meta-Lindelöf by Proposition 8.2. Now a locally separable meta-Lindelöf space is a topological sum of separable subspaces [S]. A separable, hereditarily meta-Lindelöf space is easily seen to be hereditarily Lindelöf, so the result follows. 
Proposition 8.8. Suppose $X$ is a regular, locally separable Fréchet space satisfying (1.3) or (equivalently) (1.4). Then $X$, as well as every regular pseudo-open s-image of $X$, is a topological sum of $\boldsymbol{\aleph}_{0}$-spaces.

Proof. Suppose $X$ satisfies the conditions of the proposition. By Proposition 8.6, $X$ is hereditarily meta-Lindelöf. Let $Y$ be a regular pseudo-open $s$-image of $X$. By Proposition 8.7, $Y=\Sigma_{\alpha} Y_{\alpha}$, where each $Y_{\alpha}$ is separable. By 7.1(g) and (a), each $Y_{\alpha}$ satisfies (1.4). Hence each $Y_{\alpha}$ is an $\aleph_{0}$-space by Theorem 5.2.

Note that Proposition 8.8 generalizes Theorem 5.2, which was used in its proof.

COROLlaRY 8.9. Let $X$ be a regular Fréchet space. If $X$ is determined by a point-countable collection of $\boldsymbol{\aleph}_{0}$-spaces, then $X$ is a topological sum of $\boldsymbol{\aleph}_{0}$-spaces.

Proof. Let $\mathscr{P}$ be a point-countable collection of $\boldsymbol{\aleph}_{0}$-spaces which determines $X$. Then the obvious map $f: \Sigma \mathscr{\rho} \rightarrow X$ is a quotient $s$-map by Lemma 1.8, and hence $f$ is pseudo-open because $X$ is Fréchet. Since $\Sigma \mathscr{P}$ is a regular, locally separable Fréchet space satisfying (1.4), the result now follows from Proposition 8.8.

\section{Examples.}

EXAMPLE 9.1. An infinite, completely regular, countably compact space $X$, all of whose compact subsets are finite. (This space satisfies (1.4) - even (1.6) - but not (1.3).)

Proof. Frolik [Fr] has shown that there is an infinite, countably compact subspace $X$ of $\beta \mathbf{N}$, the Stone-Čech compactification of the integers, such that every compact subset of $X$ is finite.

EXAMPLE 9.2. A paracompact Fréchet space $X$ satisfying (1.3) but not (1.1). (Hence $X$ satisfies (1.4) but not (1.6). $)^{22}$

Proof. Let $X$ be the space $S_{\omega_{1}}$, which is obtained by identifying the limit points of $\omega_{1}$ convergent sequences $X_{\alpha}\left(\alpha<\omega_{1}\right)$. This $X$ satisfies (1.3) by Theorem 7.1(i), so it remains to show that $X$ does not satisfy (1.1).

\footnotetext{
${ }^{22}$ See Example 9.6 for a Lindelöf space with these properties. See also Example 9.7.
} 
Suppose $X$ has a cover $\mathscr{P}$ satisfying (1.1); we may assume that $X \in \mathscr{P}$. Let $x$ be the non-isolated point of $X$, and let $Y_{\alpha}=X_{\alpha} \backslash\{x\}$. Let $\left(P_{n}\right)$ be an enumeration of those $P \in \mathcal{P}$ such that $x \in P$ and $P$ meets infinitely many $Y_{\alpha}$. By induction, choose $x_{n} \in P_{n} \backslash\{x\}$ such that the $x_{n}$ 's are all in different $Y_{\alpha}$ 's. Let $U=X \backslash\left\{x_{n}: n \in \omega\right\}$; clearly $U$ is open in $X$. Let $\mathscr{P}(U)=\{P \in \mathscr{P}: P \subset U\}$. If $P \in \mathscr{P}(U)$ and $x \in P$, then $P \neq P_{n}$ for any $n$, so $P$ meets only finitely many $Y_{\alpha}$. Hence st $(x, \mathscr{P}(U))$ meets only countably many $Y_{\alpha}$, and thus cannot have $x$ in its interior. Since $X$ is Fréchet, this contradicts Lemma 2.6(a) (applied to the cover $\mathcal{P}(U)$ of $U)$.

Example 9.3. A two-to-one quotient map $f: M \rightarrow Y$, with $M$ a disjoint union of compact metric spaces, and $Y$ completely regular, separable, not meta-Lindelöf, and not an $\boldsymbol{\aleph}$-space. (However, $Y$ does satisfy (1.6).)

Proof. Let $S=\{1 / n: n=1,2, \ldots\} \cup\{0\}$, and let $Y=[0,1] \times S$. Let $Y^{\prime}=[0,1] \times\{1 / n: n=1,2, \ldots\}$ have the usual Euclidean topology as a subspace of $[0,1] \times S$. Define a typical neighborhood of $(t, 0)$ in $Y$ to be of the form

$$
\{(t, 0)\} \cup\left(\bigcup_{k=n}^{\infty} U\left(t, \frac{1}{k}\right)\right),
$$

where $U(t, 1 / k)$ is a neighborhood of $(t, 1 / k)$ in $[0,1] \times\{1 / k\}$.

It is easily verified that $Y$ is completely regular.

Clearly $Y^{\prime}$ is a dense separable subspace of $Y$, and $[0,1] \times\{0\}$ is a closed discrete subspace of $Y$. Hence $Y$ is separable but not Lindelöf, and thus $Y$ is not meta-Lindelöf.

It is easy to see that $Y$ is determined by the collection

$$
\{[0,1] \times\{1 / n\}: n=1,2, \ldots\} \cup\{\{t\} \times S: t \in[0,1]\}
$$

of compact metric spaces, and each point is in at most two elements of this collection. Thus $Y$ is the two-to-one quotient image of the topological sum $M$ of these compact metric spaces, by Lemma 1.8.

Finally, $Y$ is not an $\boldsymbol{\aleph}$-space, because Foged [Fo] has shown that a $k$-and- $\aleph$-space must be meta-Lindelöf.

EXAMPLE 9.4. A regular Lindelöf space with a point-countable base which is not separable (and thus not an $\aleph_{0}$-space). 
Proof. Let $P$ be an uncountable subset of $[0,1]$ containing no uncountable closed subsets (i.e., a Bernstein set). Then the space obtained from $[0,1]$ by isolating the points of $P$ clearly satisfies the desired properties.

EXAMPLE 9.5. A separable, regular, Lindelöf $k$-space $X$ satisfying (1.1) which is not an $\boldsymbol{\aleph}_{0}$-space (or even an $\boldsymbol{\aleph}$-space).

Proof. As in Example 9.4, let $P$ be an uncountable subset of $[0,1]$ which contains no uncountable closed subsets. The space $X$ is the same as the space $Y$ of Example 9.3, except that for $t \notin P$ we define a typical neighborhood of $(t, 0)$ to be of the form

$$
U \times(\{0\} \cup\{1 / k: k \geq n\}),
$$

where $U$ is a Euclidean neighborhood of $t$ in $[0,1]$. Now $X$ is easily seen to be a regular, separable, Lindelöf $k$-space. It is not an $\boldsymbol{\aleph}_{0}$-space, since $P \times\{0\}$ is an uncountable relatively discrete subspace. It is not an $\boldsymbol{\aleph}$-space, since a Lindelöf $\boldsymbol{\aleph}$-space must be an $\boldsymbol{\aleph}_{0}$-space. It remains to show that $X$ satisfies (1.1). Let $A=\{(y, 0): y \notin P\}$. There is a countable base $\Re$ in $X$ for the points of $A$. Now $X \backslash A$ is homeomorphic to an open subspace of Example 9.3. Hence there is a point-countable collection $\mathcal{P}$ which satisfies (1.1) for $X \backslash A$. Then it is easily checked that $\Re \cup \mathcal{P}$ satisfies (1.1) for $X$.

EXAMPLE 9.6. A regular Lindelöf Fréchet space $F$ satisfying (1.3) but not (1.1).

Proof. Let $H$ be the space of Example 9.4, and let

$$
F=(H \times(\omega+1)) / H \times\{\omega\} .
$$

(Here $\omega+1$ carries the usual order topology.) Since $H \times(\omega+1)$ has a point-countable base, $F$ satisfies (1.3), by Theorem 7.1(i). But $F$ has a subspace homeomorphic to $S_{\omega_{1}}$, the space of Example 9.2 (namely $(K \times(\omega+1)) / K \times\{\omega\}$, where $K$ is a set of $\omega_{1}$ isolated points of $\left.H\right)$. Thus, by Theorem 7.1(b), $F$ does not satisfy (1.1).

EXAMPLE 9.7. A separable, regular, Lindelöf $k$-space $G$ satisfying (1.3) but not (1.1).

Proof. Let $X$ be the space of Example 9.5. Let

$$
G=X \times(\omega+1) / X \times\{\omega\} .
$$


Then $G$ satisfies (1.3) by Theorem 7.1(d) and (i). But, as in the previous example, $G$ contains a copy of Example 9.2, so $G$ does not satisfy (1.1).

EXAMPLE 9.8. A perfect map $f: X \rightarrow Y$, with $X$ a paracompact $k$-space satisfying (1.1) but with $Y$ not satisfying (1.1). (However, $Y$ must satisfy (1.3) by Theorem 7.1(e).)

Proof. Let $Z$ be the topological sum of the unit interval $[0,1]$ and the collection $\{S(x): x \in[0,1]\}$ of $2^{\omega}$ convergent sequences $S(x)$. Let $X$ be the space obtained from $Z$ by identifying the limit point of $S(x)$ with $x \in[0,1]$, for each $x \in[0,1]$. Then $X$ is a quotient $s$-image of a metric space, so $X$ is a $k$-space satisfying (1.1) by Theorem 6.1.

Let $Y$ be the space obtained from $X$ by identifying $[0,1]$ to a single point, and let $f$ be the quotient map. Clearly, $f$ is perfect. It is easy to see that $Y$ contains a closed subspace homeomorphic to the space of Example 9.2. Hence $Y$ does not satisfy (1.1).

REMARK. It is not known whether there is a Fréchet space with the properties of the space $X$ in Example 9.8.

REMARK. A separable Lindelöf space having the properties of the space $X$ of Example 9.8 can be obtained from Example 9.5 in much the same way that $X$ in Example 9.8 was obtained from Example 9.2. We omit the proof. Note that, by Theorem 5.2, this space could not be a Fréchet space.

EXAMPLE 9.9. An $\aleph_{0}$-space $X$ satisfying (1.1) which is not a $k$-space.

Proof. The space of Example 1.2 of $\left[\mathbf{M}_{3}\right]$ has the required properties. It is shown in $\left[\mathbf{M}_{3}\right]$ that this space is an $\boldsymbol{\aleph}_{0}$-space which is not a $k$-space. The proof that it satisfies (1.1) is quite long and complicated, and we omit it.

REMARK. The above example, although not a $k$-space, is a $k_{R}$-space (i.e., every real-valued function, whose restriction to every compact set is continuous, is continuous). We don't know an example of a space satisfying (1.1) which is not even a $k_{R}$-space, but conjecture that such spaces exist. 
EXAMPLE 9.10. A regular quotient space $X$ of a countable metric space, such that:

(a) $X$ has a countable cover $\mathcal{P}$ satisfying (1.1) for which the conclusion of Lemma 2.6(a) is false.

(b) $X$ has a subspace $Y$ which does not satisfy (1.3).

Proof. Let $X$ be the quotient space of the topological sum of $\Sigma_{n \in \omega} S_{n}$ of countably many convergent sequences (including their limits) $S_{n}$, obtained by identifying, for all $n>0$, the limit of $S_{n}$ with the $n$th term of $S_{0}$. (This $X$ is sometimes called $S_{2}$.)

(a) Let $\mathscr{P}$ be the set of all sets $P$ such that $P$ is a finite or cofinite subset of $S_{n}$ for some $n \in \omega$. It is easy to check that $\mathscr{P}$ satisfies (1.1) and that $x \notin(\operatorname{st}(x, \mathcal{P}))^{0}$ if $x$ is the limit of $S_{0}$.

(b) Let $Y \subset X$ consist of all isolated points of $X$, together with the limit $x$ of $S_{0}$. Suppose $\mathscr{P}$ is a cover of $Y$ satisfying (1.3) for $Y$, with $Y \in \mathscr{P}$. Since $Y$ is countable, $\mathcal{P}$ must also be countable, and we may assume $\mathscr{P}=\mathscr{\rho} *$.

Let $\left(P_{k}\right)$ be an enumeration of those $P \in \mathscr{P}$ which meet infinitely many $S_{n}$. Inductively choose $y_{k} \in P_{k} \backslash\{x\}$ such that each $y_{k}$ is in a different $S_{n}$. Let $U=Y \backslash\left\{y_{k}: k \in \omega\right\}$. Then $x \in U$, and $U$ is open in $Y$. If $P \in \mathscr{P}$ and $P \subset U$, then $P \neq P_{k}$ for any $k$; hence $P$ meets only finitely many $S_{n}$ and therefore $\{x\} \cap P$ is open in $P$. But $\{x\}$ is not open in $U$, so $U$ is not determined by $\{P \in \mathcal{P}: P \subset U\}$. Since $\mathscr{P}=\mathcal{P}^{*}$, that contradicts our hypothesis that $\mathcal{P}$ satisfies (1.3).

EXAMPLE 9.11. A countable Fréchet $\boldsymbol{\aleph}_{0}$-space $X$ such that:

(a) $X$ has a countable cover $\mathscr{P}$ satisfying (1.1) for which the conclusion of Lemma $2.6(\mathrm{~b})$ is false.

(b) $X \times Q$ does not satisfy (1.3), where $Q$ denotes the rationals.

Proof. Let $X=S_{\omega}$, the space obtained by identifying the limits of countably many convergent sequences $S_{n}$. Let $x$ be the unique non-isolated point of $X$.

(a) Let $\mathscr{T}_{n}$ be a countable base for $S_{n}$, and let $\mathscr{P}=\cup_{n} \Re_{n}$. This $\mathscr{P}$ has all the required properties, with the conclusion of Lemma 2.6(b) false at $x$.

(b) Since property (1.3) is closed hereditary, it suffices to find a closed subspace $A$ of $X \times Q$ which is homeomorphic to the space $Y$ in Example 9.10(b).

For each $n$, let $x_{n i} \rightarrow x_{n}$ be the sequence $S_{n}$ in the above definition of $X$. Let $\left(y_{n}\right)$ be a sequence of irrationals with $y_{n} \rightarrow 0$, and, for each $n$, pick 
$q_{n i} \in Q$ with $q_{n i} \rightarrow y_{n}$. Let

$$
A=\{(x, 0)\} \cup\left\{\left(x_{n i}, q_{n i}\right): n, i \in \omega\right\} .
$$

It is easy to check that the subset $A$ of $X \times Q$ has the required properties.

REMARK. Example 9.11(b) should be compared with Theorem 7.1(d), which implies that $X \times Y$ satisfies (1.3) whenever $X$ satisfies (1.3) and $Y$ is a locally compact metric space.

EXAMPLE 9.12. A space $X$ satisfying (1.2) but not (1.1). (Note: This space cannot be a $k$-space.)

Proof. The space $X$ is the ordinal space $\omega_{1}+1$, with $\omega_{1}$ having the usual neighborhoods and all points $\alpha<\omega_{1}$ isolated. The points of $X$, together with all intervals $\left(\alpha, \omega_{1}\right)$ where $\alpha<\omega_{1}$, is easily seen to be a point-countable cover of $X$ satisfying (1.2).

Suppose $\mathscr{P}$ is a point-countable cover of $X$ satisfying (1.1). Since only countably many members of $\mathscr{P}$ contain the point $\omega_{1}$, we can easily find an ordinal $\alpha<\omega_{1}$ such that, if $P \in \mathscr{P}$ and $\omega_{1} \in P \subset\left(\alpha, \omega_{1}\right]$, then $P=\left\{\omega_{1}\right\}$. Thus $P \cap\left(\alpha, \omega_{1}\right)$ is closed in $P$ for each $P \in \mathscr{P}$ with $P \subset\left(\alpha, \omega_{1}\right]$, but of course $\left(\alpha, \omega_{1}\right)$ is not closed in $\left(\alpha, \omega_{1}\right]$. Thus $X$ does not satisfy (1.1).

EXAMPLE 9.13. A sequence-covering open map between separable metric spaces which is not compact-covering.

Proof. An example of an open surjection between separable metric spaces which is not compact-covering was given in [ $\mathbf{M}_{5}$, Example 4.1]. But it is easy to check that every open surjection $f: X \rightarrow Y$ with first-countable $X$ is sequence-covering (in fact, every convergent sequence in $Y$ is the image of a convergent sequence in $X$ ), so that completes the proof.

Our next example is preceded by the following lemma. In both the lemma and the example, $S$ denotes $\{0\} \cup\{1 / n: n=1,2, \ldots\}$.

LEMMA 9.14. Let $Z$ be a separable metric space. Then there is a closed subset $Y$ of $S \times Z$ containing $\{0\} \times Z$ such that $\pi_{1}(Y)=S$ but $\pi_{1}(B) \neq S$ for any proper closed $B \subset Y$. (Here $\pi_{1}: S \times Z \rightarrow S$ denotes the coordinate projection.) 
Proof. Let $\left(S_{n}\right)$ be a disjoint cover of $S \backslash\{0\}$ by infinite subsets, and let $\left(y_{n}\right)$ be a countable dense subset of $Z$. Let

$$
Y=(\{0\} \times Z) \cup \bigcup_{n=1}^{\infty}\left(S_{n} \times\left\{y_{n}\right\}\right) .
$$

It is easy to check that $Y$ satisfies our requirements.

EXAMPLE 9.15. A quotient map $f: X \rightarrow S$, with $X$ separable metric, which is not compact-covering. Moreover, $f$ can be chosen to satisfy either of the following requirements:

(a) $f=h \circ g$, with $g$ open and $h$ perfect. $^{23}$

(b) $X$ is countable and complete.

Proof. (a) In Lemma 9.14, take $Z$ to be a closed interval. Then $Y$ is a compact metric space of cardinality $2^{\omega}$, so the proof of $\left[\mathbf{M}_{6}\right.$, Lemma 4.1] shows that there is a continuous open map $g: X \rightarrow Y$ from a separable metric space $X$ onto $Y$ which is not compact-covering. Then $f=\pi_{1} \circ g$ satisfies our requirements.

(b) In Lemma 9.14, take $Z$ to be a countable, compact metric space with infinitely many non-isolated points. Then $Y$ will also have these properties, so the proof of $\left[\mathbf{M}_{\mathbf{7}}\right.$, Theorem 1] implies that there is a quotient map $g: X \rightarrow Y$ from a countable, complete metric space onto $Y$ which is not compact covering. Then $f=\pi_{1} \circ g$ satisfies our requirements.

REMARK. Example 9.15(a) provides a negative answer to the question asked immediately after the statement of Theorem 1.4 in $\left[\mathbf{M}_{\mathbf{8}}\right]$. It follows from [ $\mathbf{M}_{\mathbf{8}}$, Theorem 1.4] that the map $f$ in Example 9.15 cannot be chosen to satisfy both conditions (a) and (b) of that example.

EXAMPLE 9.16. An open map $f: X \rightarrow Y$, with each $f^{-1}(y)$ countable, from an $\boldsymbol{\aleph}_{0}$-space $X$ onto a regular, first-countable space $Y$ which does not satisfy (1.4).

Proof. Such a map is constructed in Example 12.6 of $\left[\mathbf{M}_{1}\right]$, where it is shown that $Y$ is not an $\boldsymbol{\aleph}_{0}$-space. By Theorem 5.2, this implies that $Y$ cannot satisfy (1.4).

\footnotetext{
${ }^{23} \mathrm{~A}$. V. Ostrovskii has kindly informed us that he also obtained such an example with essentially the same proof.
} 
10. Some questions. We conclude this paper with a list of open problems, some of which have already been mentioned elsewhere in the text. See Theorem 7.1 and the examples in $\$ 9$ for some related results.

(10.1). Suppose $X$ is a quotient $s$-image of a metric space (equivalently, $X$ is a $k$-space satisfying (1.1)). Is $X$ a compact-covering $s$-image of a metric space? Does $X$ satisfy (1.6)? (An affirmative answser to the second question implies an affirmative answer to the first.) (See Footnote 10 and remarks preceding and following Theorem 6.1.)

(10.2). Are pseudo-open $s$-images of metric spaces (equivalently, Fréchet spaces satisfying (1.1)) preserved by pseudo-open $s$-maps? By perfect maps? (See Example 9.8 and remarks following Corollary 6.2).

(10.3). Are spaces (or $k$-spaces) satisfying (1.1) preserved by open $s$-maps? (The answer is positive for Fréchet spaces; see Theorem 7.1(f)).

(10.4). Are $k$-spaces satisfying (1.3) (equivalently, (1.4)) preserved by quotient $s$-maps? By pseudo-open $s$-maps? By open $s$-maps? (Without the $k$-space assumption, (1.4) need not be preserved by open $s$-maps; see Example 9.16.)

(10.5). Are spaces satisfying (1.3) preserved by perfect maps? (The answer is positive for $k$-spaces, since in this case (1.3) and (1.4) are equivalent-see Theorem 7.1(e)).

(10.6). Is the pseudo-open $s$-image of a meta-Lindelöf (or even paracompact) space meta-Lindelöf? Is the closed image of a meta-Lindelöf space meta-Lindelöf? (See the remark following Proposition 8.2.)

(10.7). Does every closed image of a metric space satisfy (1.3) (equivalently, (1.4))? (By Example 9.2, it need not satisfy (1.1).)

(10.8). Is every space satisfying (1.1) a $k_{R}$-space? (See the remark following Example 9.9.)

(10.9). Is property $(1.3)_{p}$ preserved by arbitrary subsets? By finite or countable products?

(10.10). Does Proposition 3.2 remain true without assuming that $X$ has countable tightness?

\section{REFERENCES}

[A] A. V. Arhangel'skiur, Some types of factor mappings and the relations between classes of topological spaces, Dokl. Akad. Nauk. SSSR, 153 (1963), 743-746. (Soviet Math. Dokl. 4 (1963), 1726-1729.)

[Ba] Z. Balogh, On relative countable compactness, Uspekhi Mat. Nauk, 34 : 6 (1979), 139-143.

$\left[\mathrm{BM}_{1}\right]$ D. Burke and E. Michael, On a theorem of $V$. V. Filippov, Israel J. Math., 11 (1972), 394-397. 
$\left[\mathrm{BM}_{2}\right]$ D. Burke and E. Michael, On certain point-countable covers, Pacific J. Math., 64 (1976), 79-92.

[Fo] L. Foged, personal communication.

[Fr] Z. Frolik, Generalizations of the $G_{\delta}$-property of complete metric spaces, Czech. Math. J., 10 (1960), 359-379.

[H] T. Hoshina, On the quotient s-images of metric spaces, Sci. Rep. Tokyo Kyoiku Daigaku Sect. A, 10 (1970), 265-268.

[J] H. Junnila, Paracompactness, metacompactness, and semi-open covers, Proc. Amer. Math. Soc., 73 (1979), 244-248.

$\left[\mathrm{M}_{1}\right] \quad$ E. Michael, $\boldsymbol{\aleph}_{0}$-spaces, J. Math. Mech., 15 (1966), 983-1002.

$\left[\mathrm{M}_{2}\right]$ _ A quintuple quotient quest, General Topology and Appl., 2 (1972), 91-138.

$\left[\mathrm{M}_{3}\right] \quad \ldots$ On $k$-spaces, $k_{R}$-spaces, and $k(X)$, Pacific J. Math., 47 (1973), 487-498.

$\left[\mathrm{M}_{4}\right] \quad$ A note on closed maps and compact sets, Israel J. Math., 2 (1964), $173-176$.

$\left[\mathrm{M}_{5}\right] \ldots \aleph_{0}^{\prime}$-spaces and a function space theorem of $R$. Pol, Indiana Univ. Math. J., 26 (1977), 299-306.

$\left[\mathrm{M}_{6}\right] \quad \ldots$ A theorem on semi-continuous set-valued functions, Duke Math. J., 26 (1959), 647-652.

$\left[\mathrm{M}_{7}\right] \quad$, Quotients of countable, complete metric spaces, Proc. Amer. Math. Soc., 57 (1976), 371-372.

$\left[\mathrm{M}_{8}\right] \quad \ldots$, Inductively perfect maps and tri-quotient maps, Proc. Amer. Math. Soc., 82 (1981), 115-119.

[MN] E. Michael and K. Nagami, Compact-covering images of metric spaces, Proc. Amer. Math. Soc., 37 (1973), 260-266.

[N] K. Nagami, 2 -spaces, Fund. Math., 65 (1969), 169-192.

[O] P. O'Meara, On paracompactness in function spaces with the compact-open topology, Proc. Amer. Math. Soc., 29 (1971), 183-189.

[P] V. I. Ponomarev, Axioms of countability and continuous mappings, Bull. Acad. Polon. Sci. Sér. Math. Astronom. Phys., 8 (1960), 127-134. (Russian)

[S] A. H. Stone, Absolute $F_{\sigma}$-spaces, Proc. Amer. Math. Soc., 13 (1962), 495-499.

$\left[\mathrm{T}_{1}\right] \quad$ Y. Tanaka, Closed maps on metric spaces, Topology Appl., 11 (1980), 87-92.

$\left[\mathrm{T}_{2}\right] \quad$, Products of spaces of countable tightness, Topology Proceedings, 6 (1981), $115-133$.

[W] J. H. C. Whitehead, A note on a theorem of Borsuk, Bull. Amer. Math. Soc., 54 (1958), 1125-1132.

Received August 18, 1982. The second author was supported, in part, by a grant from the National Science Foundation.

AUBURN UNIVERSITY

AUBURN, AL 36830

UNIVERSITY OF WASHINGTON

SeAtTle, WA 98195

TOKYO GAKUGEI UNIVERSITY

(184) KoganeISHI, TOKYO, JAPAN 


\section{PACIFIC JOURNAL OF MATHEMATICS \\ EDITORS}

Donald BaBBITT (Managing Editor)

University of California

Los Angeles, CA 90024

Hugo RossI

University of Utah

Salt Lake City, UT 84112

C. C. Moore and Arthur Ogus

University of California

Berkeley, CA 94720
J. DugundiI

Department of Mathematics

University of Southern California

Los Angeles, CA 90089-1113

R. FINN and H. SAMELSON

Stanford University

Stanford, CA 94305

\section{ASSOCIATE EDITORS}
R. ARENS
E. F. BECKENBACH
B. H. NeUMANN
F. WOLF
K. YoshidA (1906-1982)

\section{SUPPORTING INSTITUTIONS}

\author{
UNIVERSITY OF ARIZONA \\ UNIVERSITY OF BRITISH COLUMBIA \\ CALIFORNIA INSTITUTE OF TECHNOLOGY \\ UNIVERSITY OF CALIFORNIA \\ MONTANA STATE UNIVERSITY \\ UNIVERSITY OF NEVADA, RENO \\ NEW MEXICO STATE UNIVERSITY \\ OREGON STATE UNIVERSITY
}

\author{
UNIVERSITY OF OREGON \\ UNIVERSITY OF SOUTHERN CALIFORNIA \\ STANFORD UNIVERSITY \\ UNIVERSITY OF HAWAII \\ UNIVERSITY OF TOKYO \\ UNIVERSITY OF UTAH \\ WASHINGTON STATE UNIVERSITY \\ UNIVERSITY OF WASHINGTON
}

The Supporting Institutions listed above contribute to the cost of publication of this Journal, but they are not owners or publishers and have no responsibility for its content or policies.

Mathematical papers intended for publication in the Pacific Journal of Mathematics should be in typed form or offset-reproduced (not dittoed), double spaced with large margins. Please do not use built up fractions in the text of the manuscript. However, you may use them in the displayed equations. Underline Greek letters in red, German in green, and script in blue. The first paragraph must be capable of being used separately as a synopsis of the entire paper. In particular it should contain no bibliographic references. Please propose a heading for the odd numbered pages of less than 35 characters. Manuscripts, in triplicate, may be sent to any one of the editors. Please classify according to the scheme of Math. Reviews, Index to Vol. 39. Supply name and address of author to whom proofs should be sent. All other communications should be addressed to the managing editor, or Elaine Barth, University of California, Los Angeles, California 90024.

There are page-charges associated with articles appearing in the Pacific Journal of Mathematics. These charges are expected to be paid by the author's University, Government Agency or Company. If the author or authors do not have access to such Institutional support these charges are waived. Single authors will receive 50 free reprints; joint authors will receive a total of 100 free reprints. Additional copies may be obtained at cost in multiples of 50 .

The Pacific Journal of Mathematics is issued monthly as of January 1966. Regular subscription rate: $\$ 132.00$ a year (6 Vol., 12 issues). Special rate: $\$ 66.00$ a year to individual members of supporting institutions.

Subscriptions, orders for numbers issued in the last three calendar years, and changes of address should be sent to Pacific Journal of Mathematics, P.O. Box 969, Carmel Valley, CA 93924, U.S.A. Old back numbers obtainable from Kraus Periodicals Co., Route 100, Millwood, NY 10546.

The Pacific Journal of Mathematics ISSN 0030-8730 is published monthly by the Pacific Journal of Mathematics at P.O. Box 969, Carmel Valley, CA 93924. Application to mail at Second-class postage rates is pending at Carmel Valley, California, and additional mailing offices. Postmaster: Send address changes to Pacific Journal of Mathematics, P. O. Box 969, Carmel Valley, CA 93924.

PUBLISHED BY PACIFIC JOURNAL OF MATHEMATICS, A NON-PROFIT CORPORATION

Copyright $\odot 1984$ by Pacific Journal of Mathematics 


\section{Pacific Journal of Mathematics}

\section{Vol. 113, No. $2 \quad$ April, 1984}

Alan Adolphson, On the Dwork trace formula ...................257

Amos Altshuler and Leon Steinberg, Enumeration of the quasisimplicial

3 -spheres and 4-polytopes with eight vertices .................. 269

Kenneth R. Goodearl, Cancellation of low-rank vector bundles .......... 289

Gary Fred Gruenhage, Ernest A. Michael and Yoshio Tanaka, Spaces

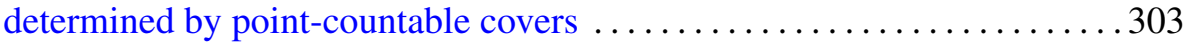

Charles Lemuel Hagopian, Atriodic homogeneous continua .......... 333

David Harbater, Ordinary and supersingular covers in characteristic $p$. . . 349

Domingo Antonio Herrero, Continuity of spectral functions and the lakes

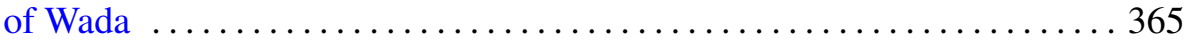

Donald William Kahn, Differentiable approximations to homotopy resolutions and framed cobordism ....................... 373

K. McGovern, On the lifting theory of finite groups of Lie type $\ldots \ldots \ldots . \ldots 383$

C. David (Carl) Minda, The modulus of a doubly connected region and the geodesic curvature-area method ............................. 395

Takuo Miwa, Complexes are spaces with a $\sigma$-almost locally finite base $\ldots . .407$

Ho Kuen Ng, Finitely presented dimension of commutative rings and modules

Roger David Nussbaum, A folk theorem in the spectral theory of

$C_{0}$-semigroups

J. S. Okon, Prime divisors, analytic spread and filtrations

Harold Raymond Parks, Regularity of solutions to elliptic isoperimetric problems

R. Sitaramachandra Rao and M. V. Subba Rao, Transformation formulae for multiple series

Daniel Ruberman, Imbedding punctured lens spaces and connected sums

Uri Srebro, Deficiencies of immersions 